for

\title{
Quantifying the Electronic and Steric Properties of 1,3- Imidazole-Based Mesoionic Carbenes (iMICs)
}

Arne Merschel, Dennis Rottschäfer, Beate Neumann, Hans-Georg Stammler, and Rajendra S. Ghadwal*

Anorganische Molekülchemie und Katalyse, Lehrstuhl für Anorganische Chemie und Strukturchemie, Centrum für Molekulare Materialien, Fakultät für Chemie, Universität Bielefeld, Universitätsstr. 25, 33615 Bielefeld, Germany.

E-mail: rghadwal@uni-bielefeld.de 


\section{Contents}

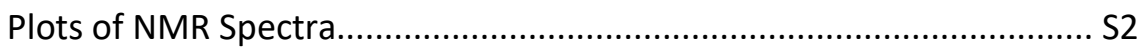

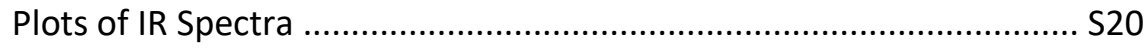

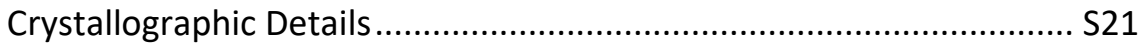

Steric Maps of (iMIC ${ }^{\mathrm{DMP}}$ ) Cul (8a) and (iMIC $\left.{ }^{\mathrm{DMP}}\right) \mathrm{CuBr}(\mathbf{8 b})$................... S24

Correlation plots of ${ }^{31} \mathrm{P}$ vs. ${ }^{77}$ Se NMR chemical shifts.......................... S25

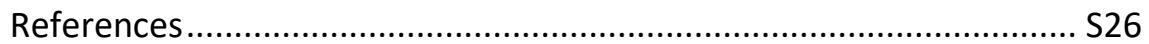




\section{Plots of NMR Spectra}
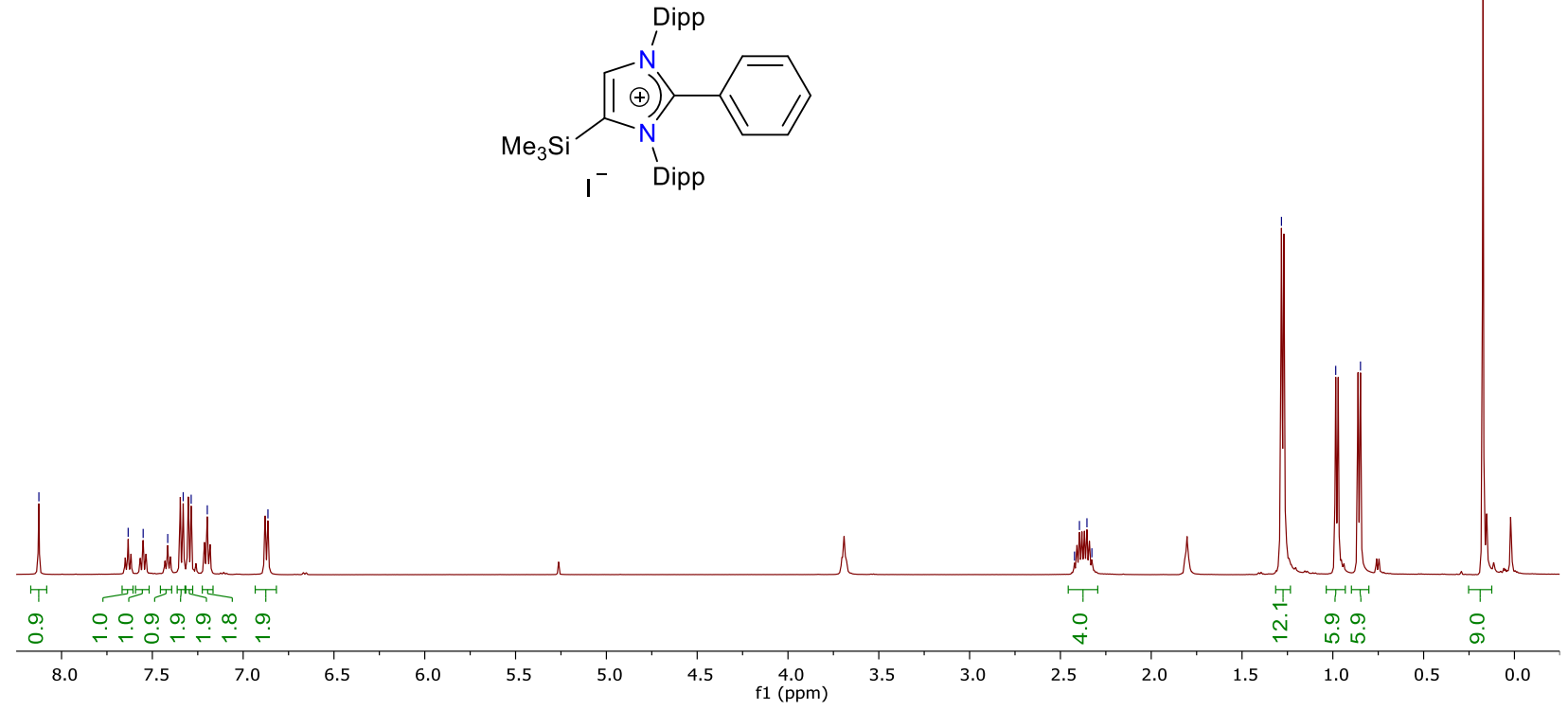

Figure S1. ${ }^{1} \mathrm{H} \mathrm{NMR}\left(500 \mathrm{MHz}, \mathrm{CDCl}_{3}, 298 \mathrm{~K}\right)$ spectrum of 3a.
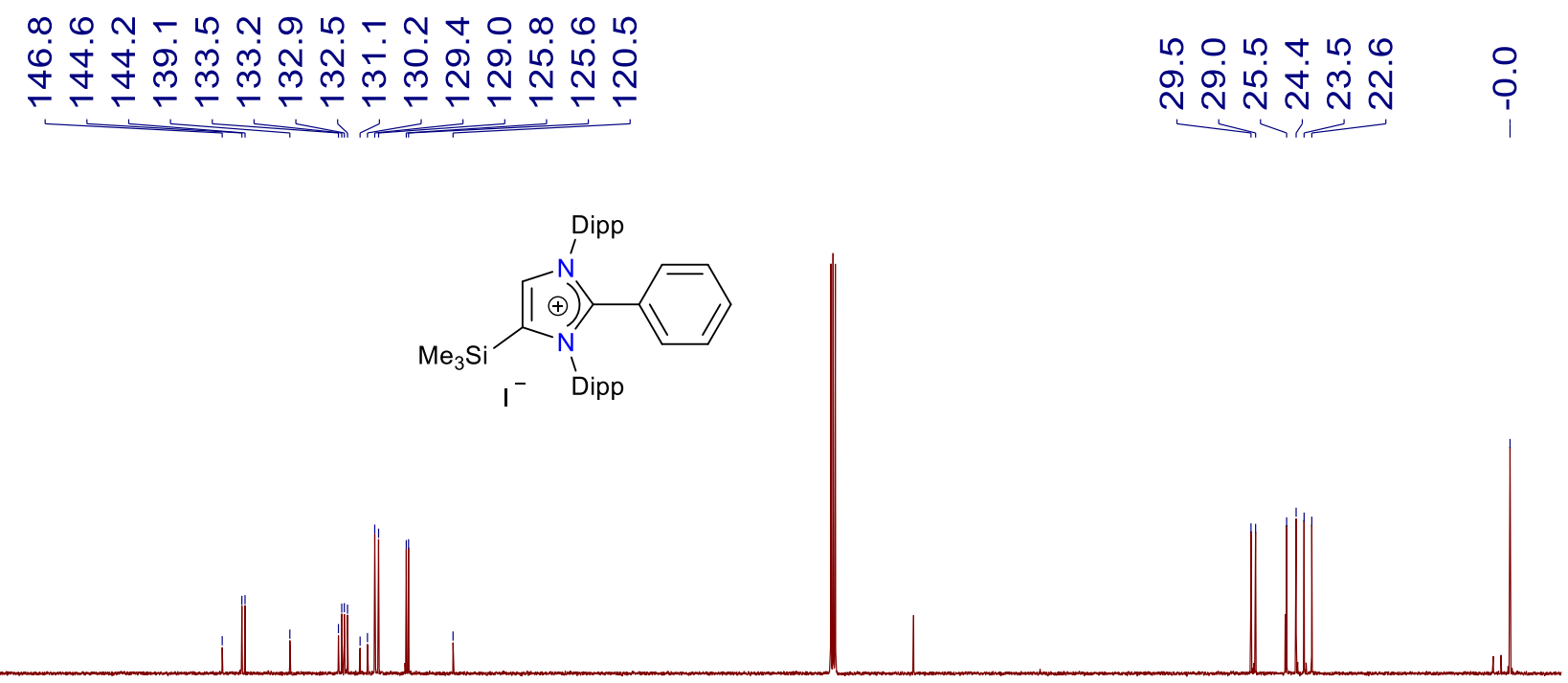

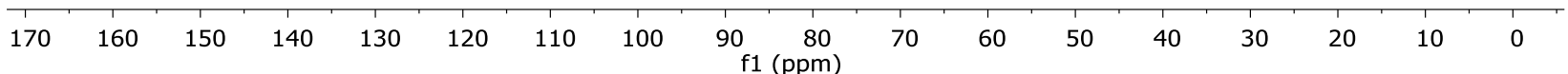

Figure S2. ${ }^{13} \mathrm{C}\left\{{ }^{1} \mathrm{H}\right\} \mathrm{NMR}\left(126 \mathrm{MHz}, \mathrm{CDCl}_{3}, 298 \mathrm{~K}\right)$ spectrum of $\mathbf{3 a}$. 


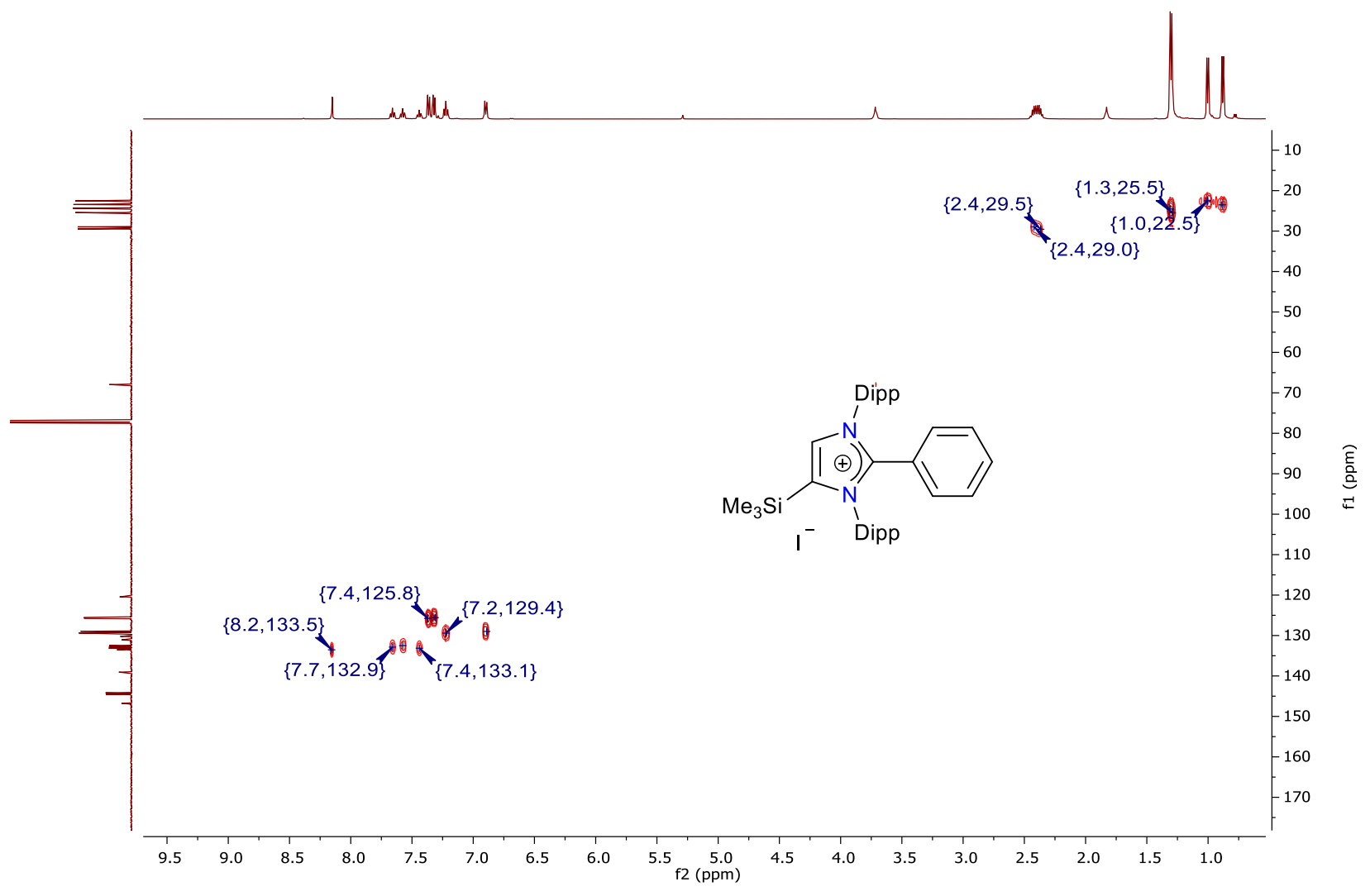

Figure S3. ${ }^{1} \mathrm{H}-{ }^{13} \mathrm{C}$ HMQC NMR (500/126 MHz, $\mathrm{CDCl}_{3}$, $\left.298 \mathrm{~K}\right)$ spectrum of 3a.

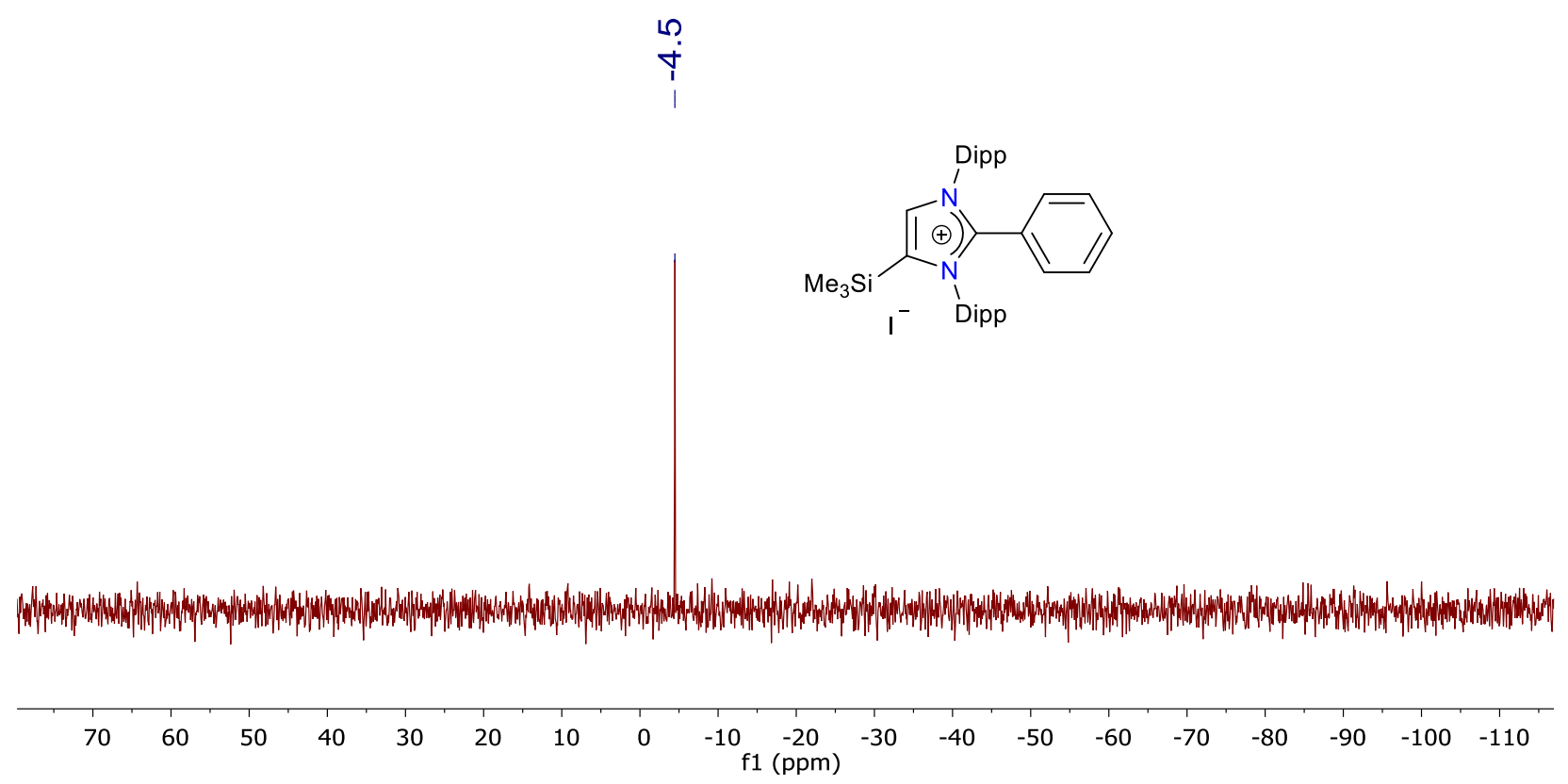

Figure S4. ${ }^{29} \mathrm{Si}\left\{{ }^{1} \mathrm{H}\right\}$ NMR (99 $\left.\mathrm{MHz}, \mathrm{CDCl}_{3}, 298 \mathrm{~K}\right)$ spectrum of 3a. 


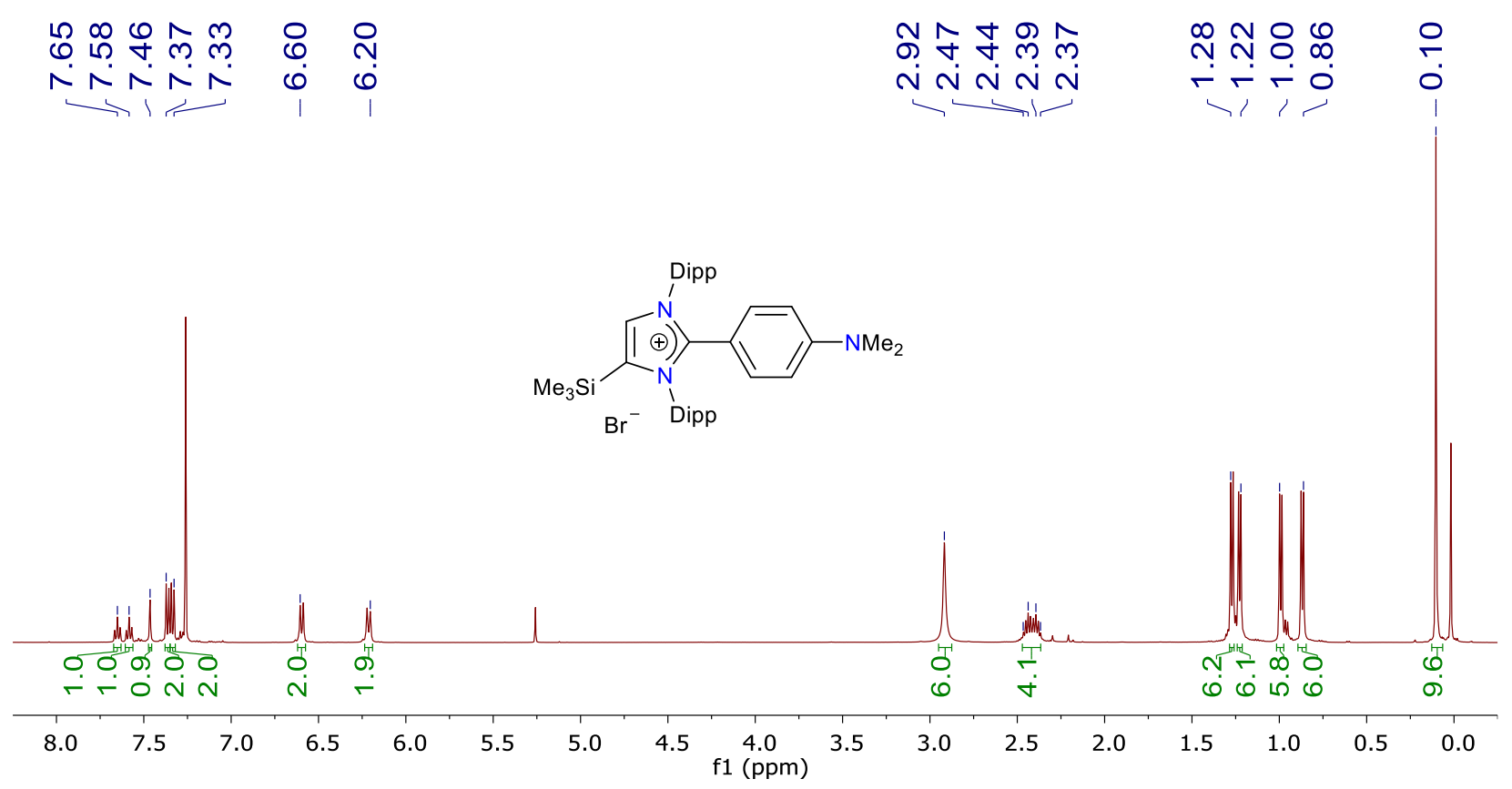

Figure S5. ${ }^{1} \mathrm{H} \mathrm{NMR}\left(500 \mathrm{MHz}, \mathrm{CDCl}_{3}, 298 \mathrm{~K}\right)$ spectrum of $\mathbf{3 b}$.

a) m m L

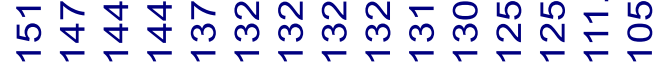
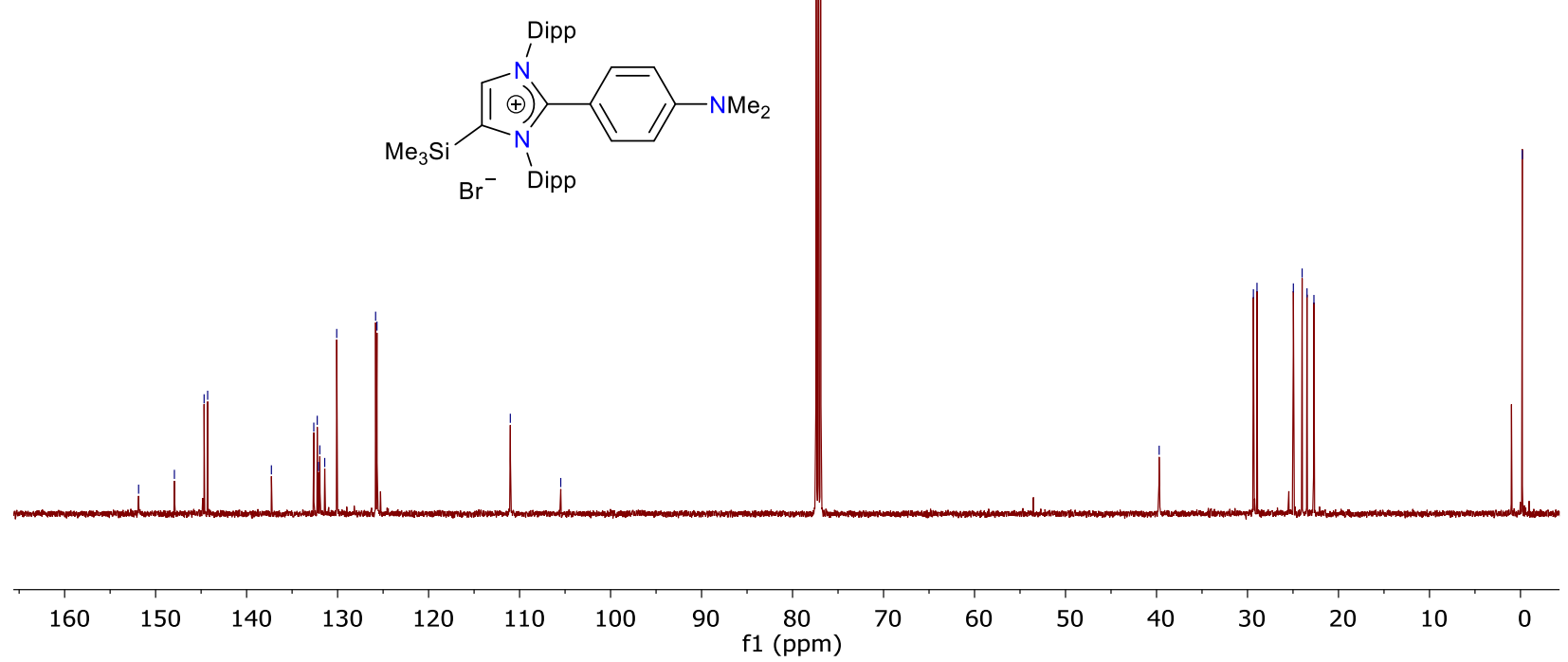

$\operatorname{tin} 0.0010$

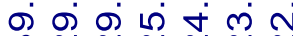
M N N N N $\stackrel{N}{0}$

Figure S6. ${ }^{13} \mathrm{C}\left\{{ }^{1} \mathrm{H}\right\}$ NMR $\left(126 \mathrm{MHz}, \mathrm{CDCl}_{3}, 298 \mathrm{~K}\right)$ spectrum of $\mathbf{3 b}$. 


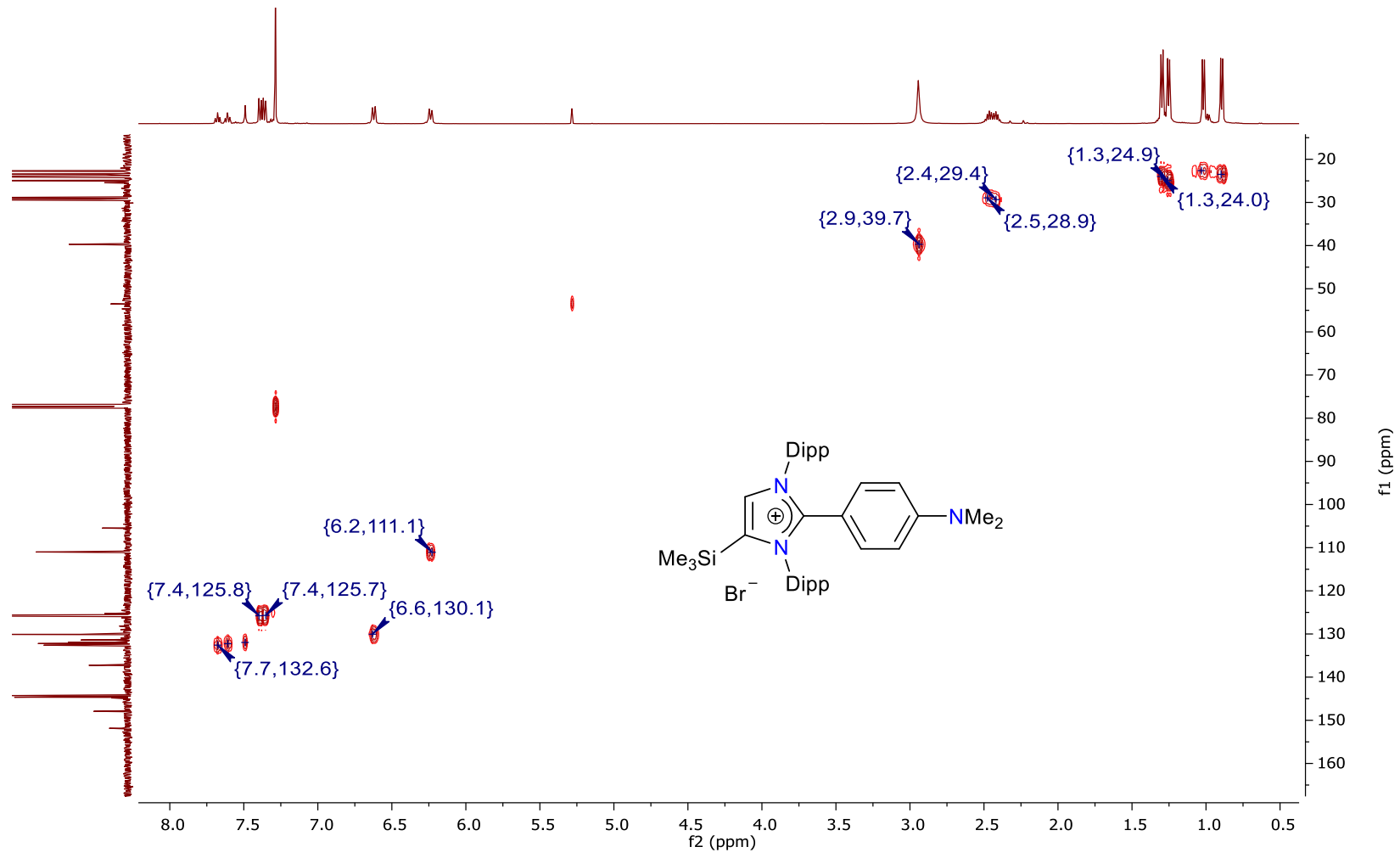

Figure S7. ${ }^{1} \mathrm{H}_{-}{ }^{13} \mathrm{C}$ HMQC NMR $\left(500 / 126 \mathrm{MHz}, \mathrm{CDCl}_{3}, 298 \mathrm{~K}\right)$ spectrum of $\mathbf{3 b}$.

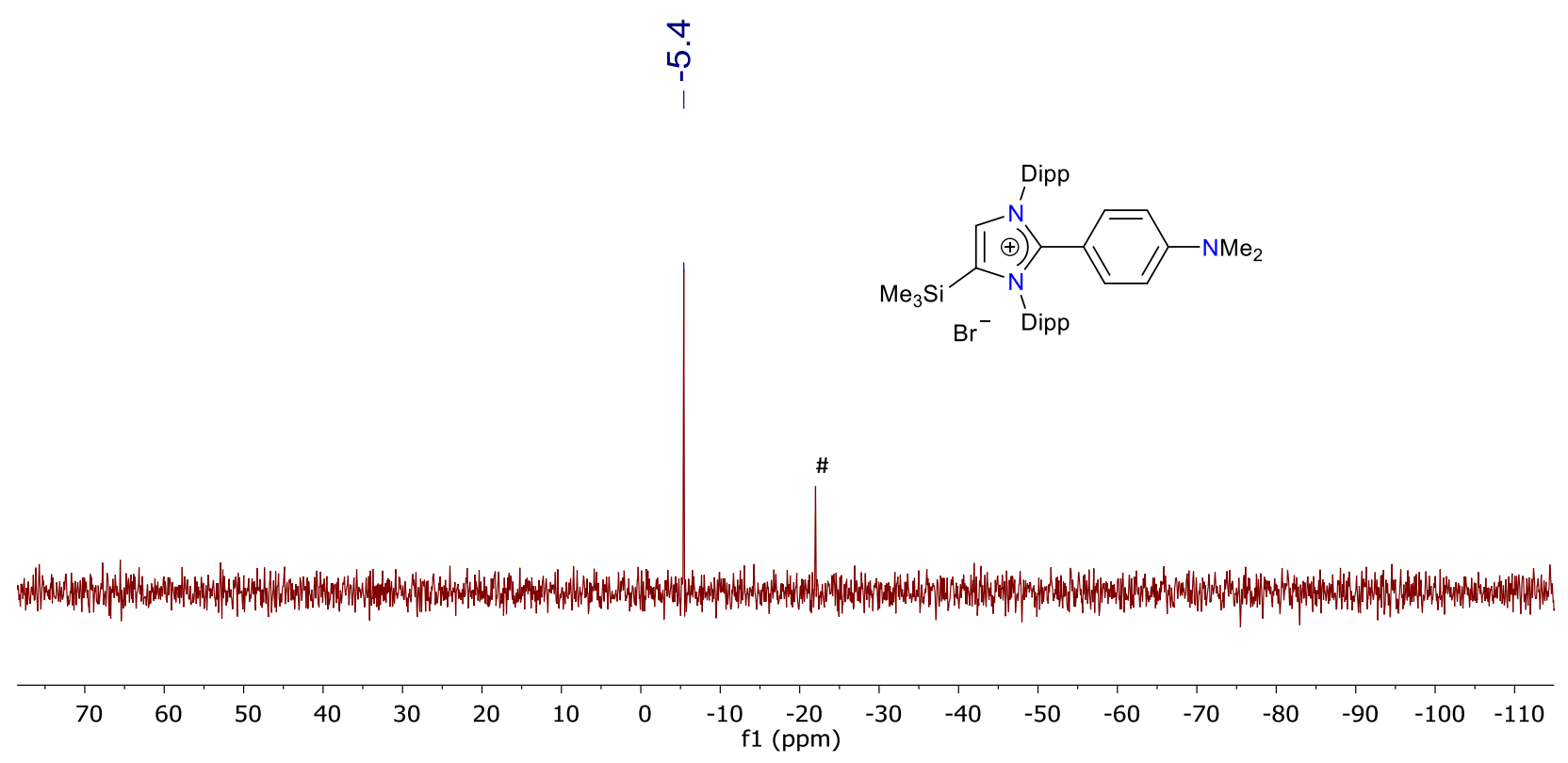

Figure S8. ${ }^{29} \mathrm{Si}\left\{{ }^{1} \mathrm{H}\right\} \mathrm{NMR}\left(99 \mathrm{MHz}, \mathrm{CDCl}_{3}, 298 \mathrm{~K}\right)$ spectrum of $\mathbf{3 b}$ ( ${ }^{\#}$ silicon grease). 


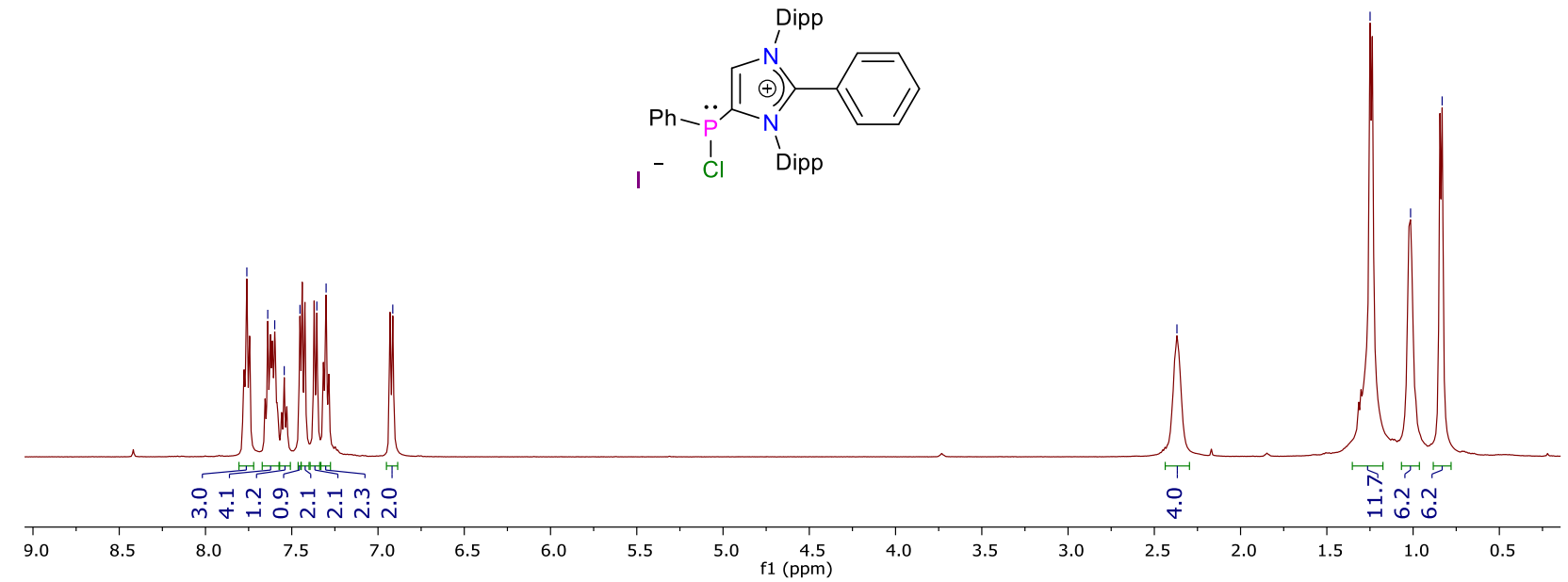

Figure S9. ${ }^{1} \mathrm{H}$ NMR $\left(500 \mathrm{MHz}, \mathrm{CDCl}_{3}, 298 \mathrm{~K}\right)$ spectrum of $\mathbf{4 a}$.

$$
\text { กิ }
$$<smiles></smiles>

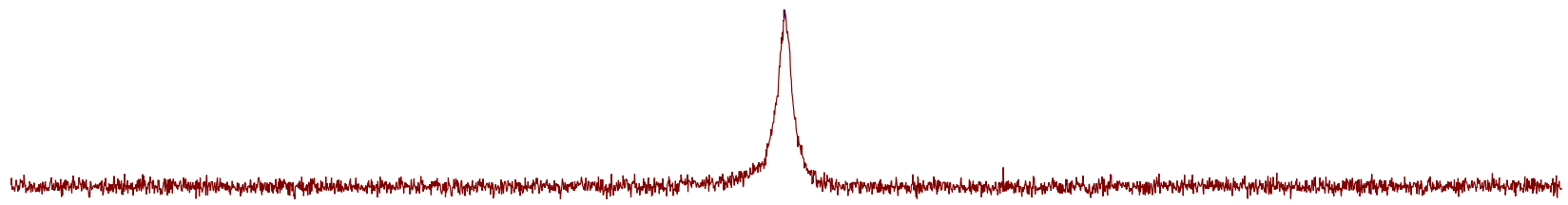

$\begin{array}{llllllllllllllllllllllllllllllllllll}105 & 100 & 95 & 90 & 85 & 80 & 75 & 70 & 65 & 60 & 55 & 50 & 45 & 40 & 35 & 30 & 25 & 20 & 15 & 10 & 5 & 0 & -5 & -10 & -15 & -20 & -25\end{array}$

Figure S10. ${ }^{31} \mathrm{P}\left\{{ }^{1} \mathrm{H}\right\} \mathrm{NMR}\left(202 \mathrm{MHz}, \mathrm{CDCl}_{3}, 298 \mathrm{~K}\right)$ spectrum of $4 \mathbf{a}$. 


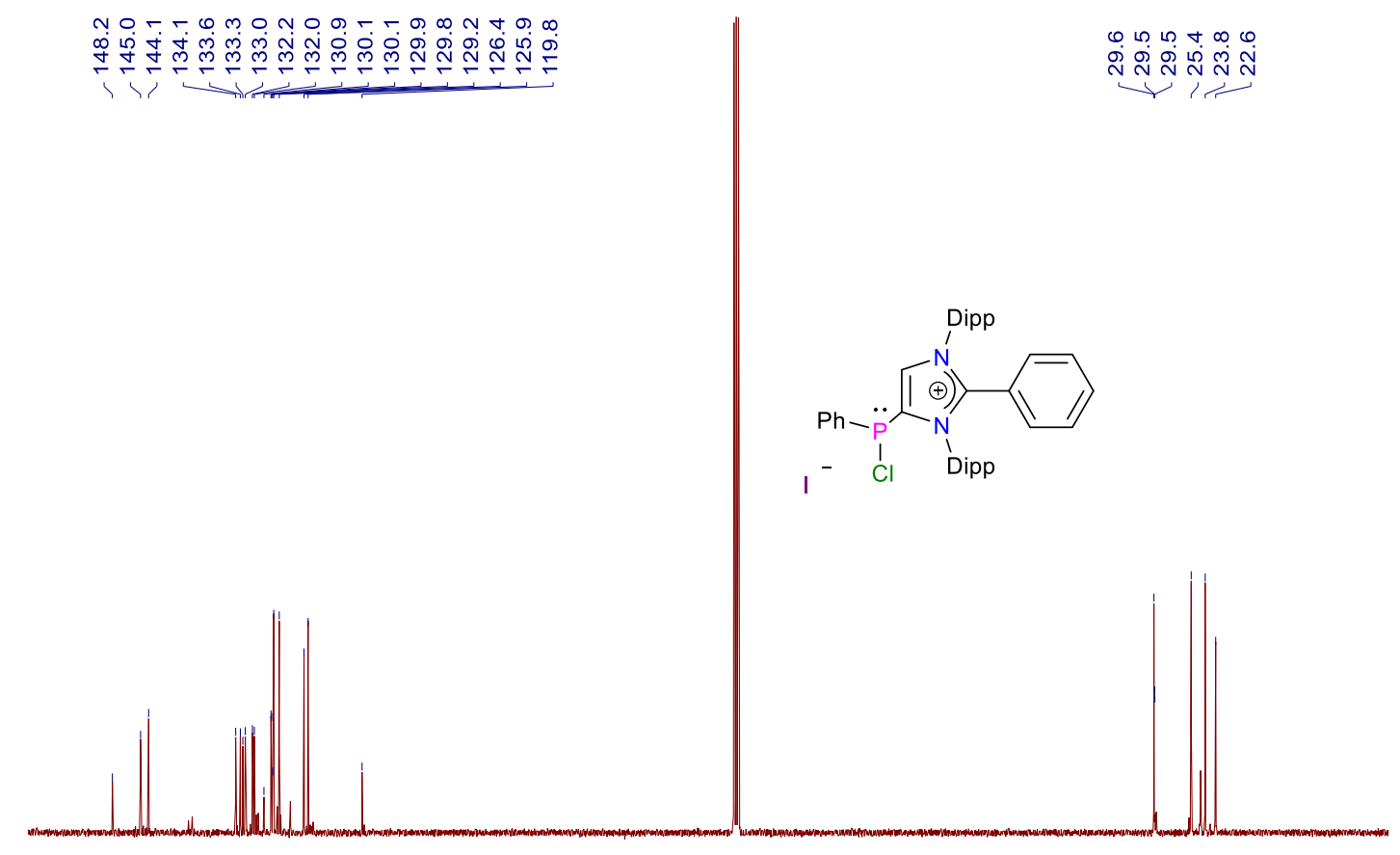

$\begin{array}{llllllllllllllllllllllllllllllllllllllllllllll}155 & 150 & 145 & 140 & 135 & 130 & 125 & 120 & 115 & 110 & 105 & 100 & 95 & 90 & 85 & 80 & 75 & 70 & 65 & 60 & 55 & 50 & 45 & 40 & 35 & 30 & 25 & 20 & 15 & 10 & 5\end{array}$

Figure S11. ${ }^{13} \mathrm{C}\left\{{ }^{1} \mathrm{H}\right\} \mathrm{NMR}\left(126 \mathrm{MHz}, \mathrm{CDCl}_{3}, 298 \mathrm{~K}\right)$ spectrum of $4 \mathbf{a}$.

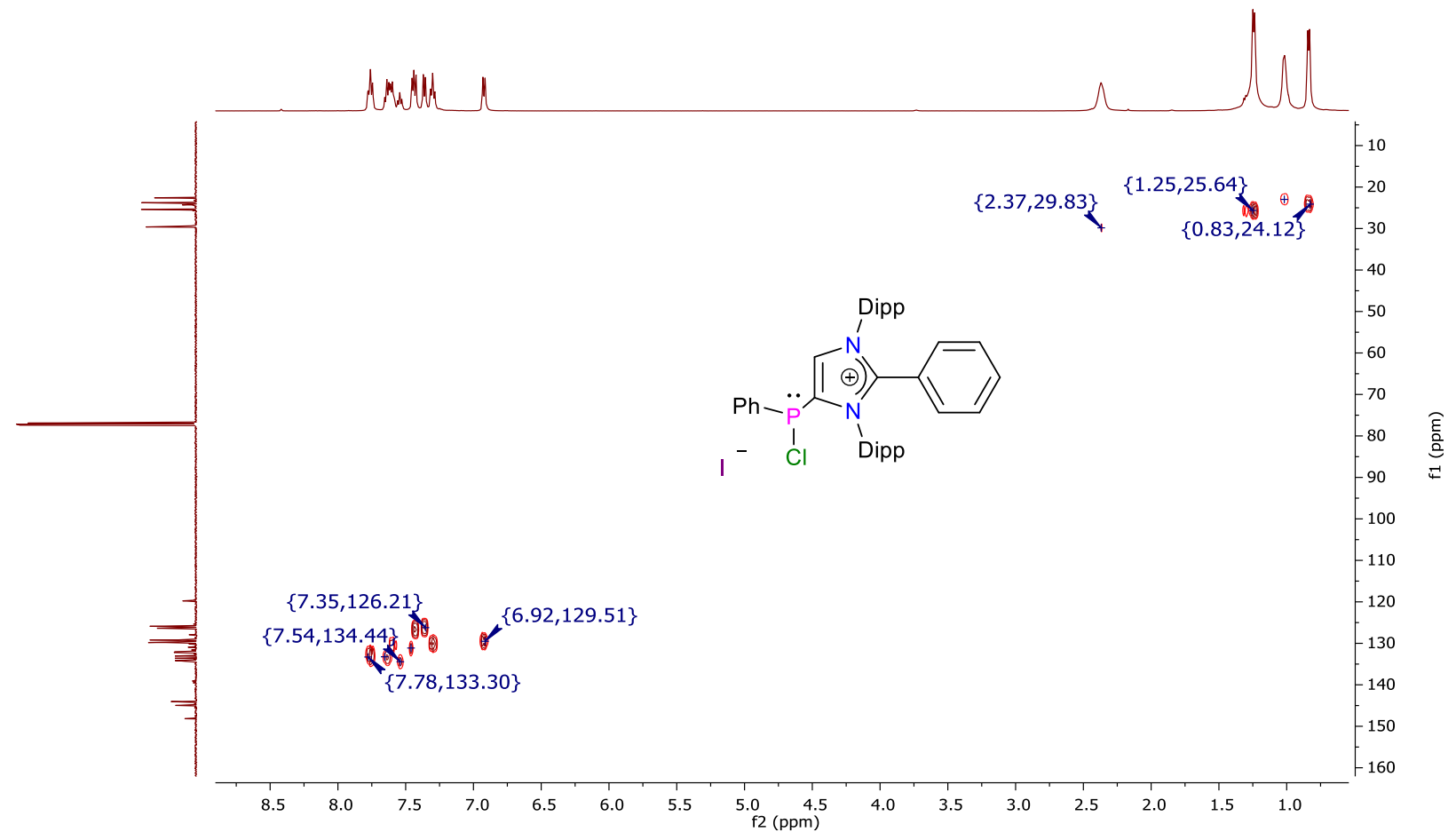

Figure S12. ${ }^{1} \mathrm{H}-{ }^{13} \mathrm{C}$ HMQC NMR $\left(500 / 126 \mathrm{MHz}, \mathrm{CDCl}_{3}, 298 \mathrm{~K}\right)$ spectrum of $4 \mathbf{a}$. 

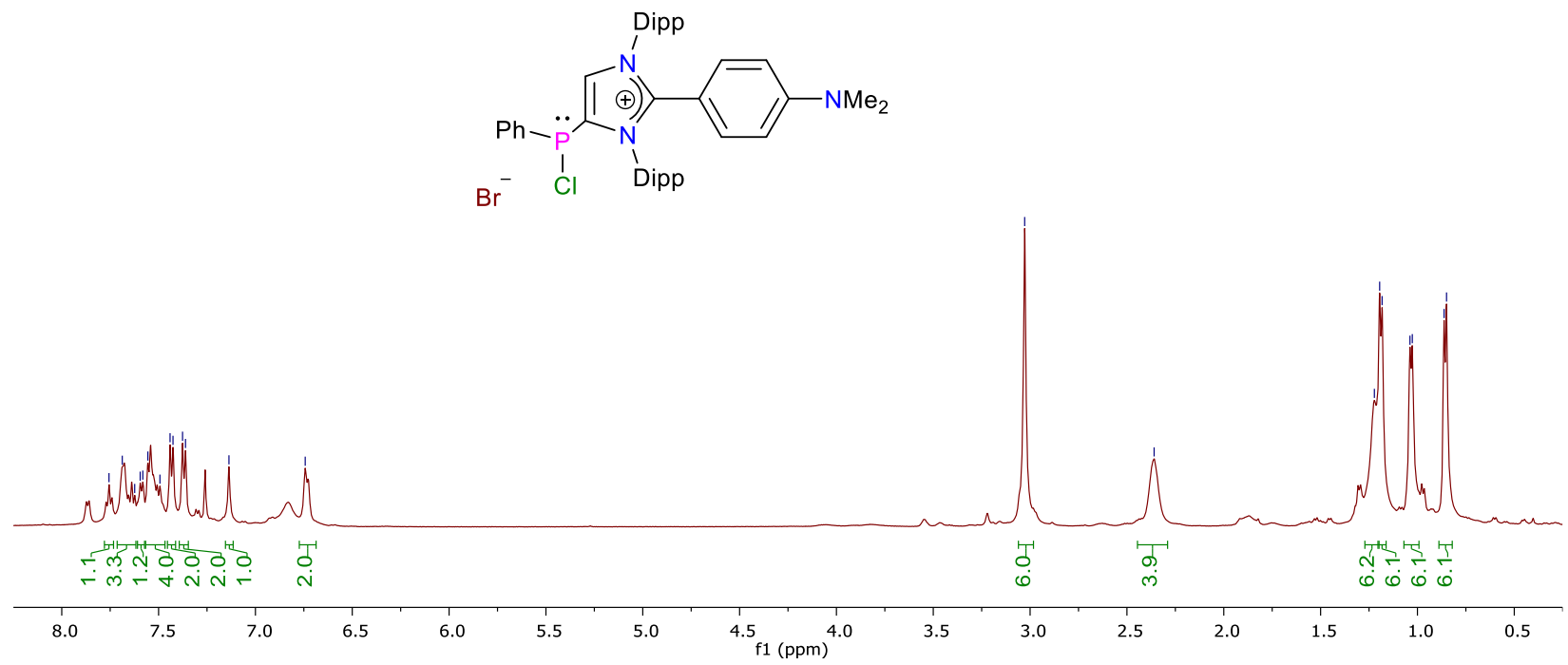

Figure S13. ${ }^{1} \mathrm{H}$ NMR $\left(500 \mathrm{MHz}, \mathrm{CDCl}_{3}, 298 \mathrm{~K}\right)$ spectrum of $\mathbf{4 b}$.
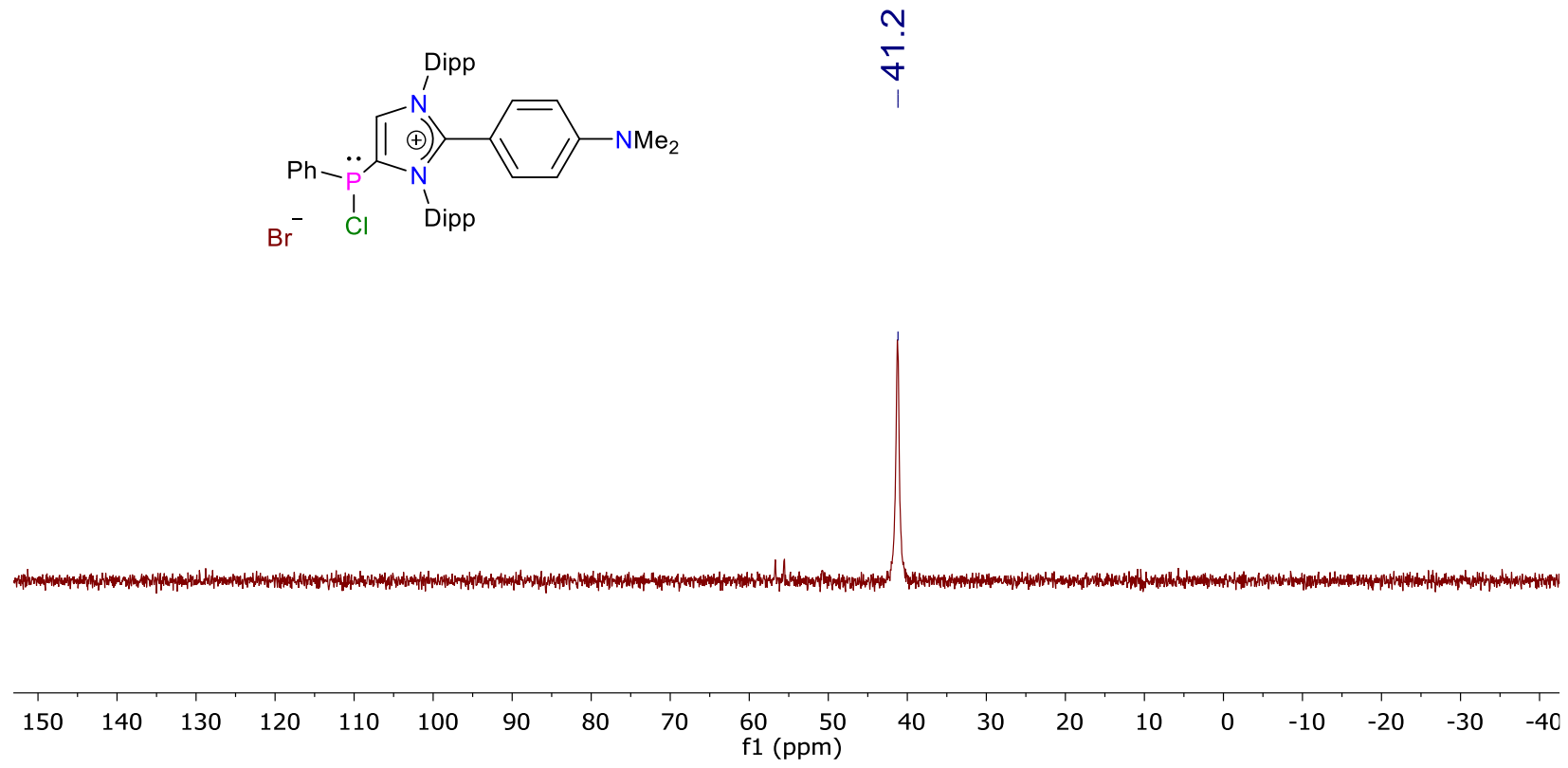

Figure S14. ${ }^{31} \mathrm{P}\left\{{ }^{1} \mathrm{H}\right\} \mathrm{NMR}\left(202 \mathrm{MHz}, \mathrm{CDCl}_{3}, 298 \mathrm{~K}\right)$ spectrum of $\mathbf{4 b}$. 

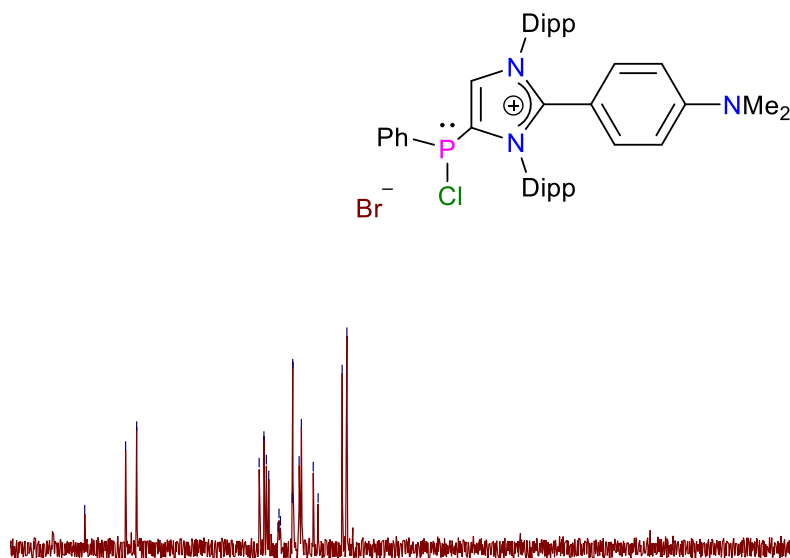

$\begin{array}{llllllllllllllll}55 & 150 & 145 & 140 & 135 & 130 & 125 & 120 & 115 & 110 & 105 & 100 & 95 & 90 & 85 & 80\end{array}$

Figure S15. ${ }^{13} \mathrm{C}\left\{{ }^{1} \mathrm{H}\right\}$ NMR $\left(126 \mathrm{MHz}, \mathrm{CDCl}_{3}, 298 \mathrm{~K}\right)$ spectrum of $\mathbf{4 b}$.

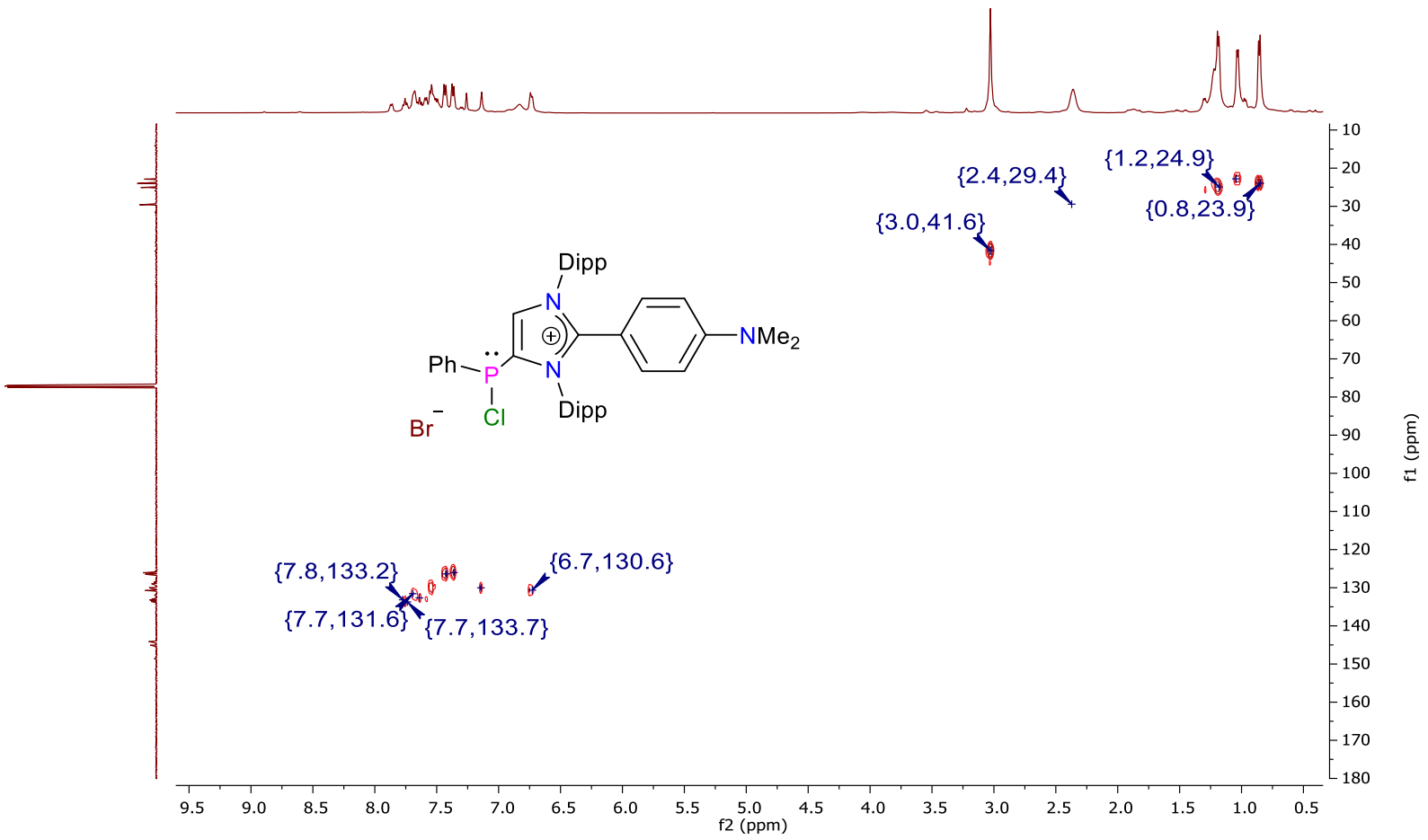

Figure S16. ${ }^{1} \mathrm{H}-{ }^{13} \mathrm{C}$ HMQC NMR $\left(500 / 126 \mathrm{MHz}, \mathrm{CDCl}_{3}, 298 \mathrm{~K}\right)$ spectrum of $\mathbf{4 b}$. 

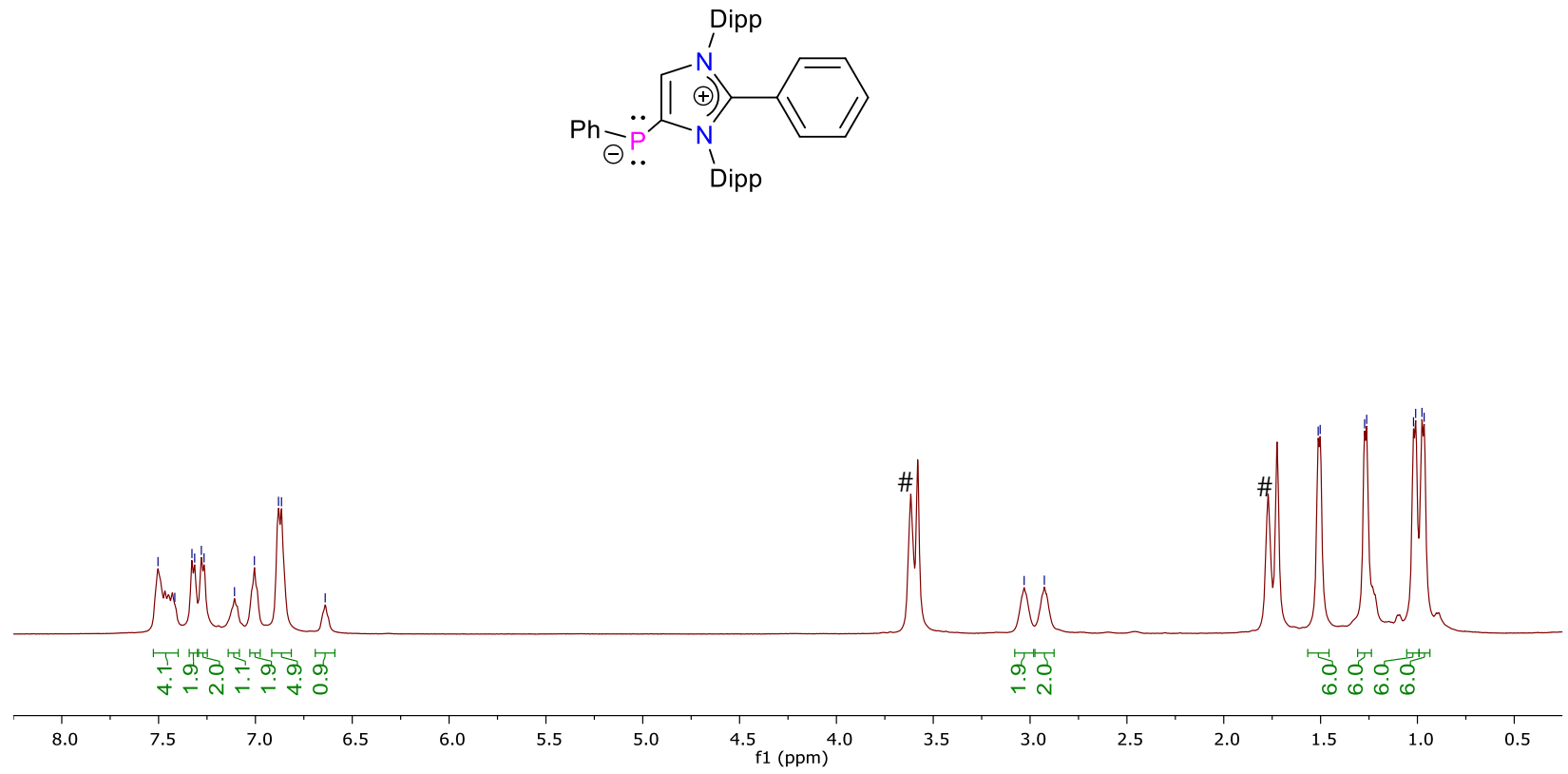

Figure S17. ${ }^{1} \mathrm{H}$ NMR $\left(500 \mathrm{MHz}, \mathrm{THF}-d_{8}, 298 \mathrm{~K}\right)$ spectrum of 5a. (\# THF)

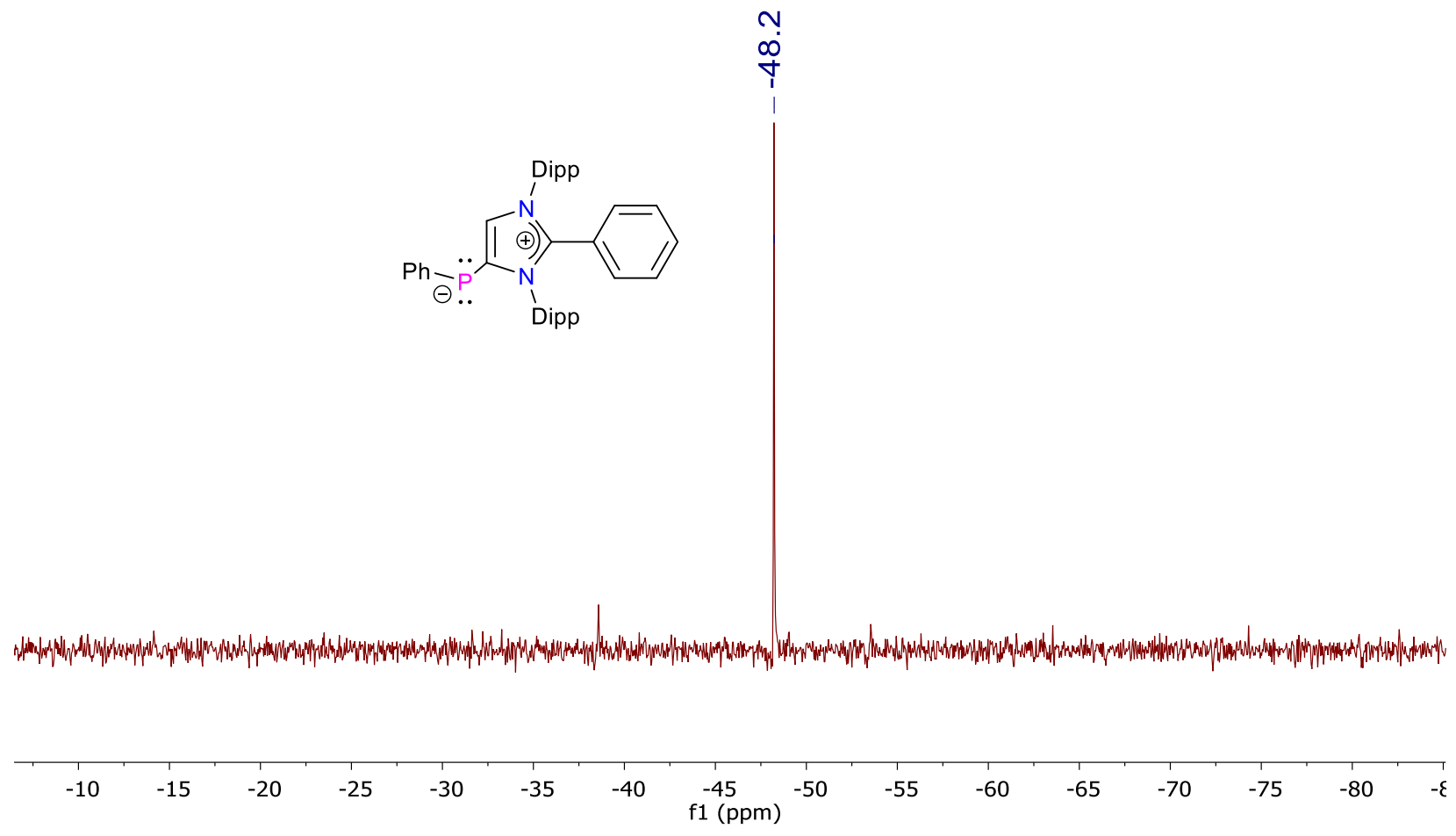

Figure S18. ${ }^{31} \mathrm{P}\left\{{ }^{1} \mathrm{H}\right\}$ NMR (202 MHz, Benzene- $\left.d_{6}, 298 \mathrm{~K}\right)$ spectrum of 5a. 


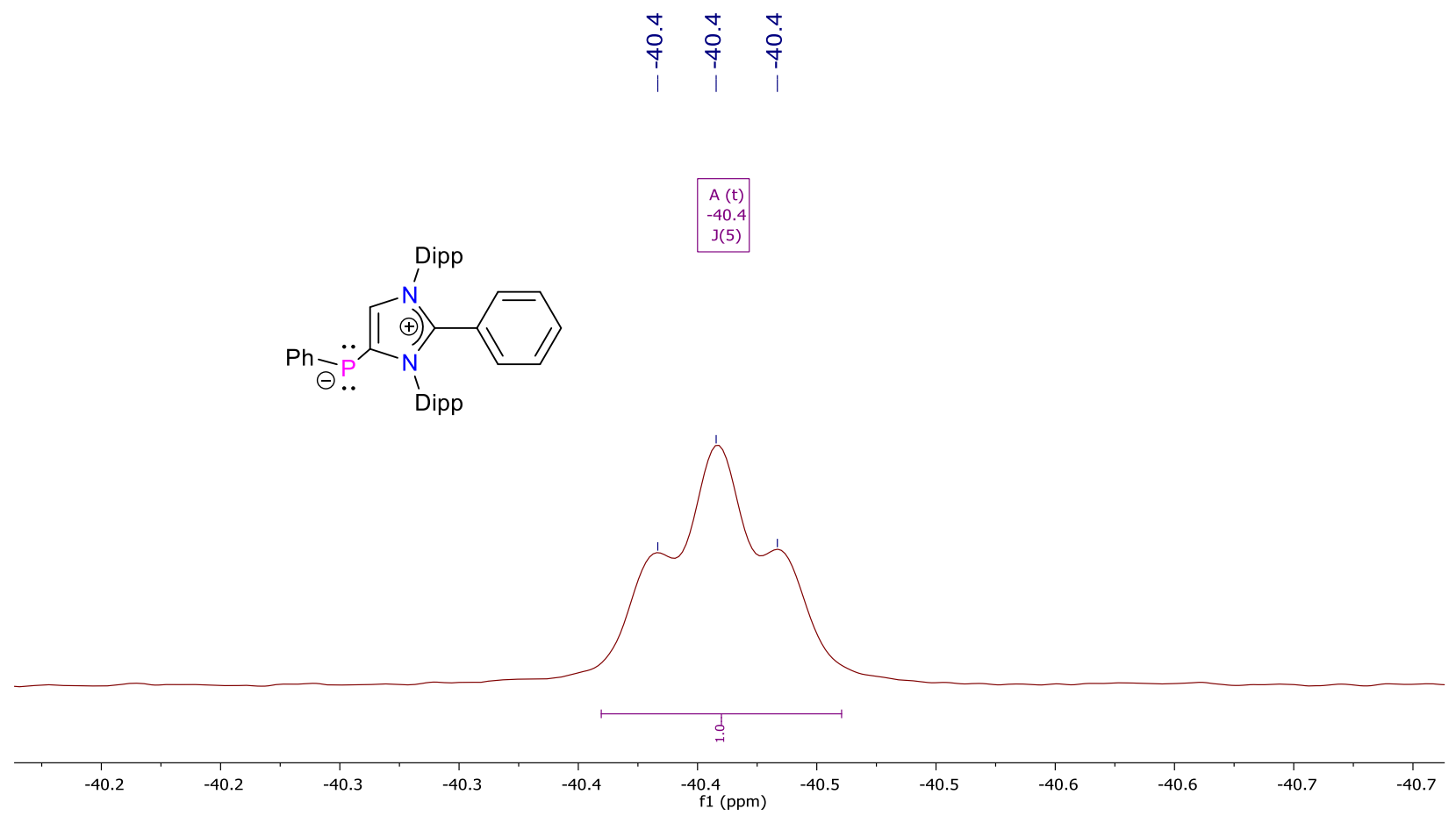

Figure S19. ${ }^{31} \mathrm{P}$ NMR $\left(202 \mathrm{MHz}\right.$, THF- $\left.d_{8}, 298 \mathrm{~K}\right)$ spectrum of $5 \mathbf{a}$.
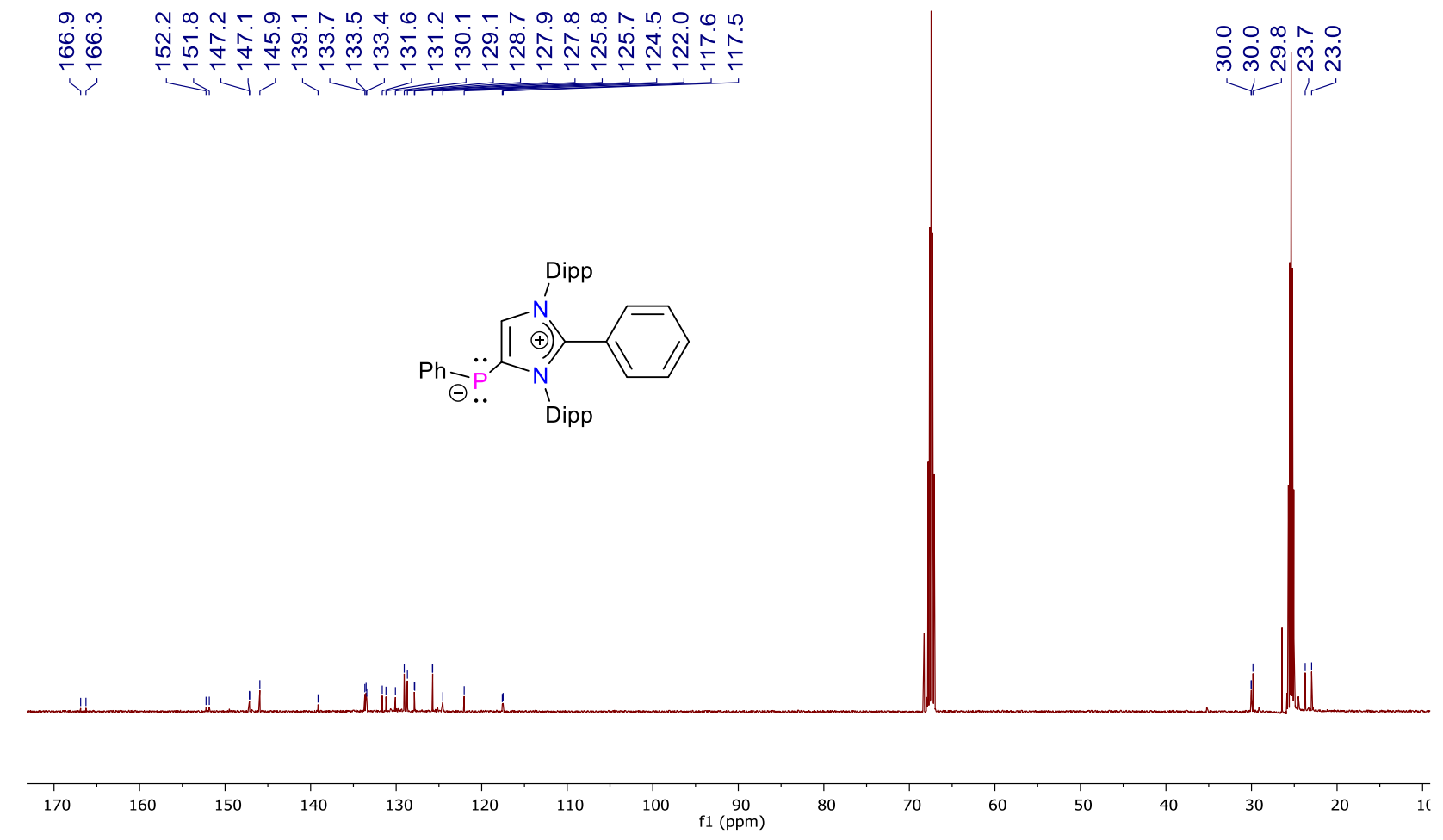

Figure S20. ${ }^{13} \mathrm{C}\left\{{ }^{1} \mathrm{H}\right\}$ NMR $\left(126 \mathrm{MHz}, \mathrm{THF}-d_{8}, 298 \mathrm{~K}\right)$ spectrum of $5 \mathbf{a}$. 


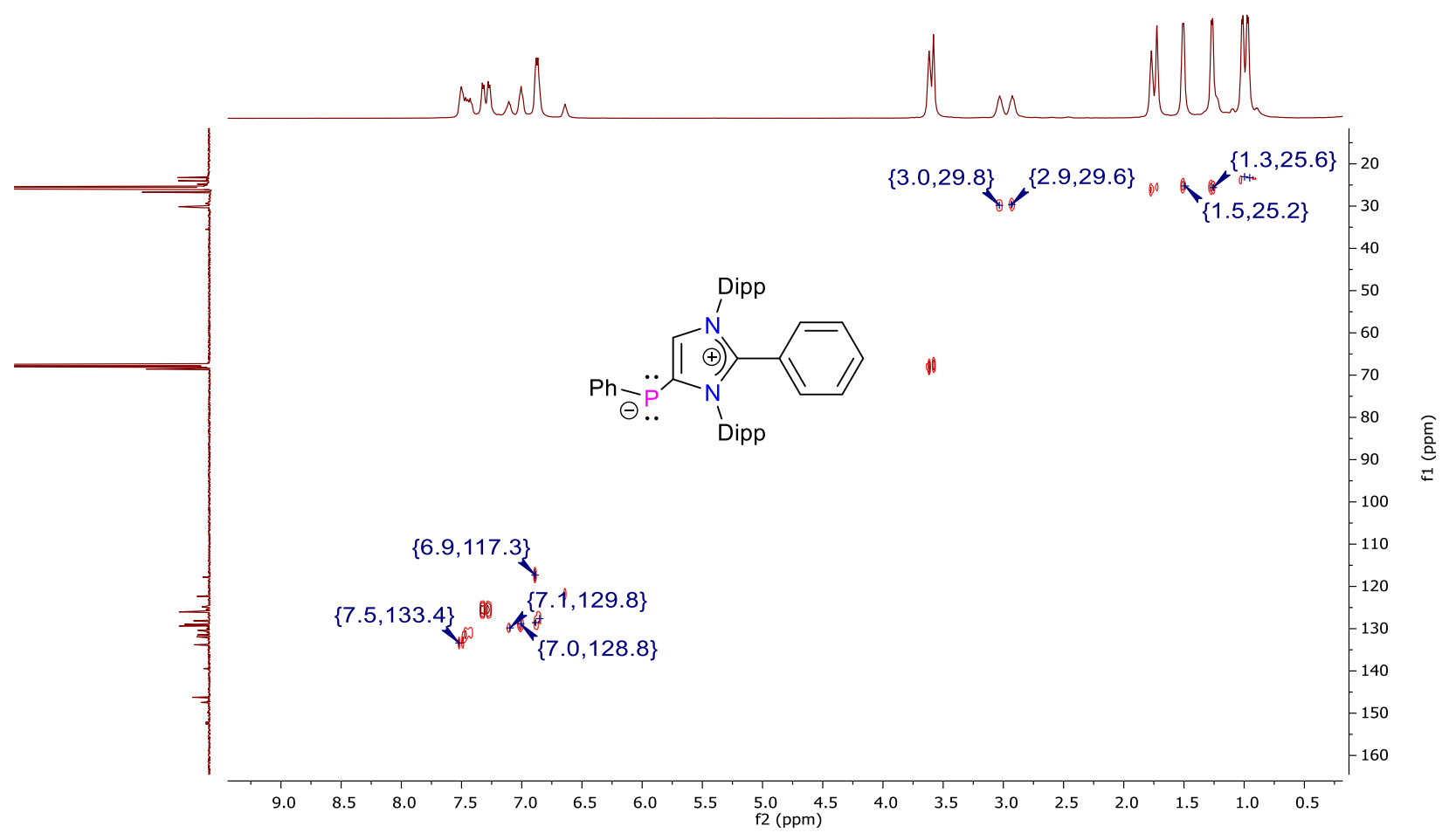

Figure S21. ${ }^{1} \mathrm{H}-{ }^{13} \mathrm{C}$ HMQC NMR $\left(500 / 126 \mathrm{MHz}\right.$, THF- $\left.d_{8}, 298 \mathrm{~K}\right)$ spectrum of $\mathbf{5 a}$.

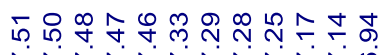

inisinñño

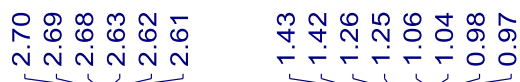

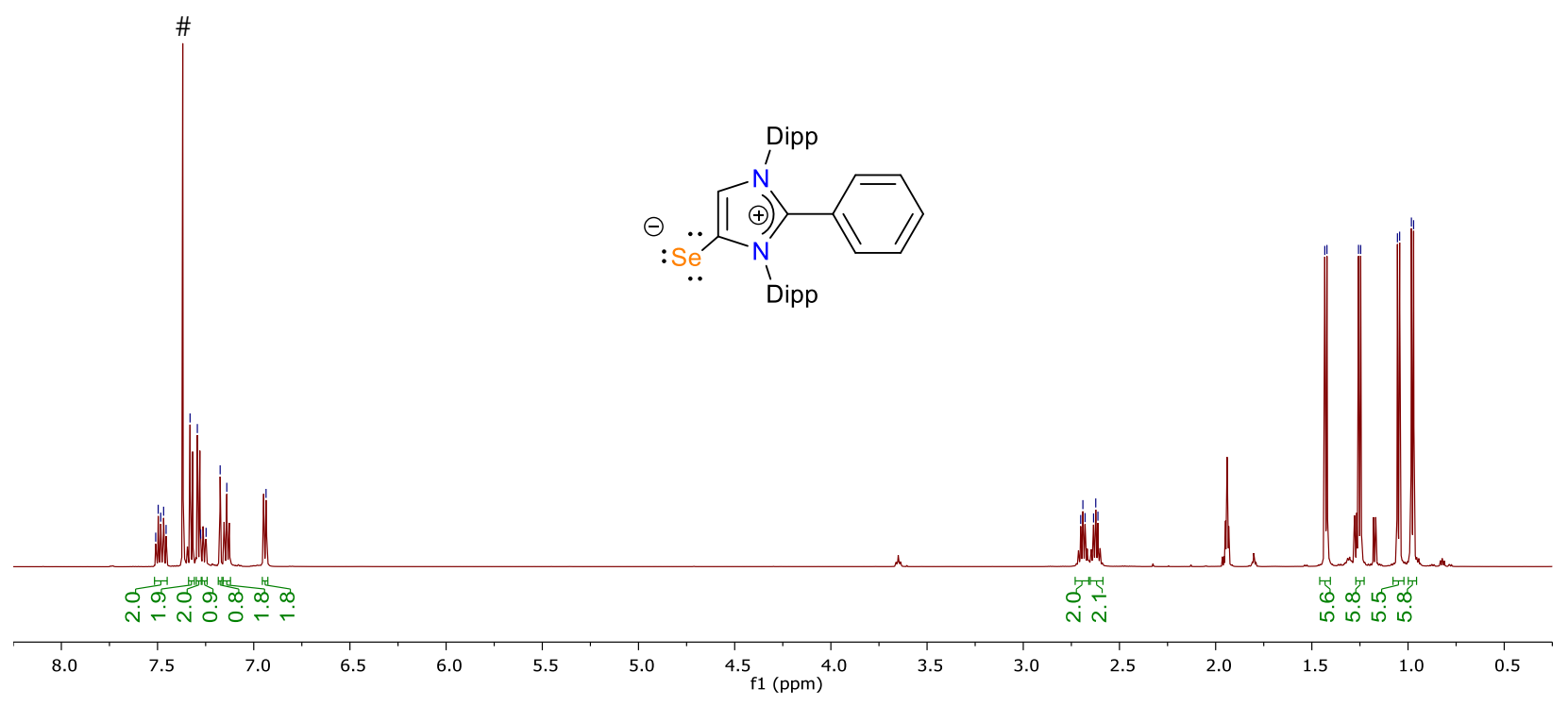

Figure S22. ${ }^{1} \mathrm{H}$ NMR (600 MHz, Acetonitrile- $\left.d_{3}, 298 \mathrm{~K}\right)$ spectrum of $6 \mathbf{6}$. (\# benzene) 


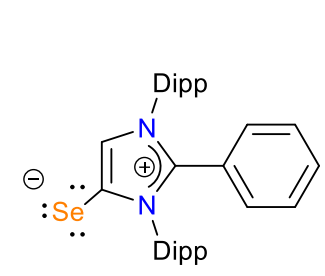

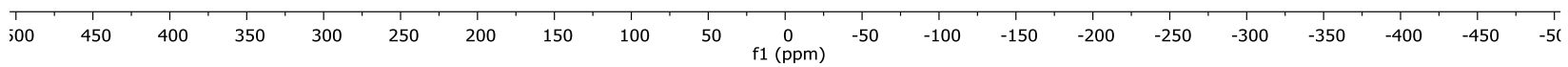

Figure S23. ${ }^{77} \mathrm{Se}\left\{{ }^{1} \mathrm{H}\right\}$ NMR (95 MHz, Acetonitrile- $\left.d_{3}, 298 \mathrm{~K}\right)$ spectrum of $6 \mathbf{a}$.

N.

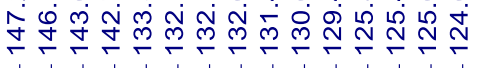

$-\infty \infty m m$

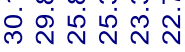

il

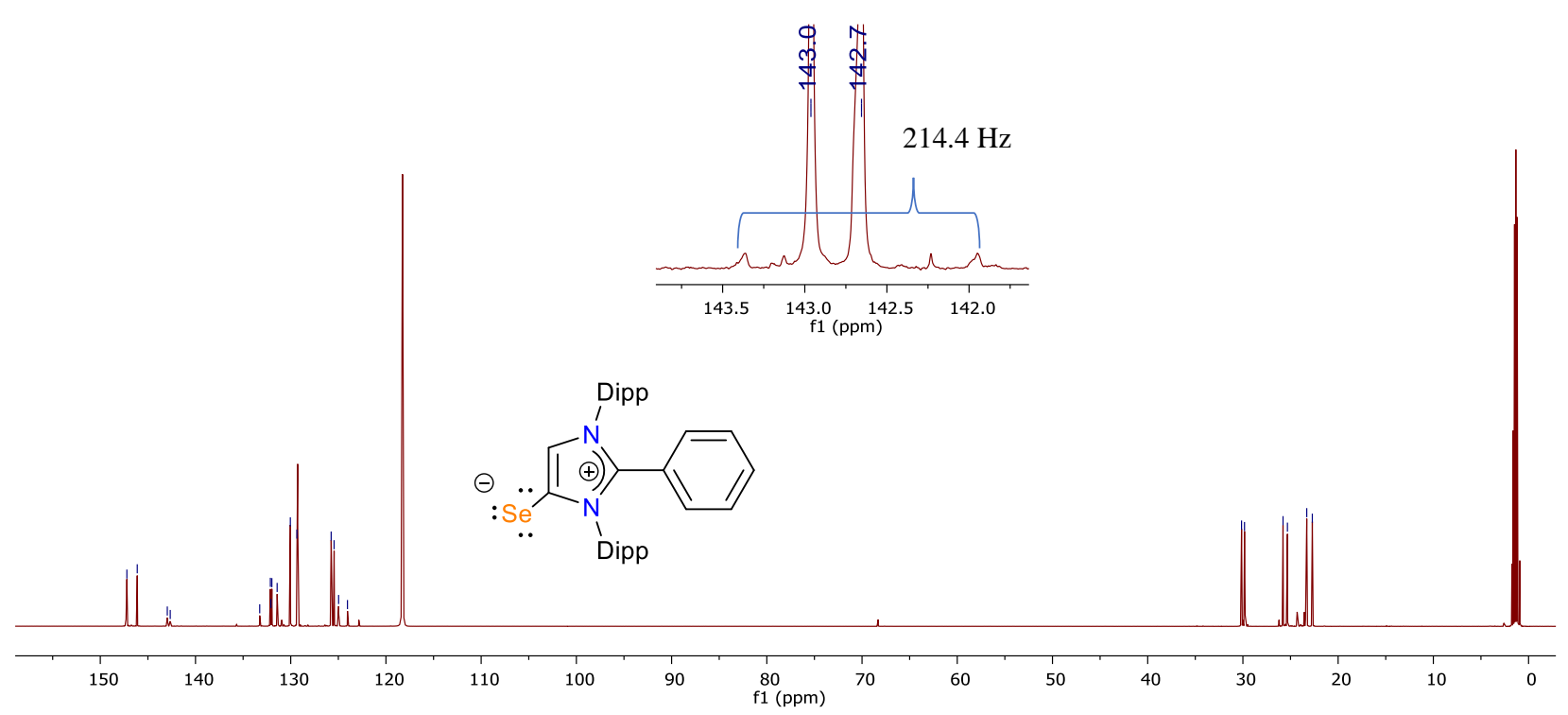

Figure S24. ${ }^{13} \mathrm{C}\left\{{ }^{1} \mathrm{H}\right\}$ NMR (151 MHz, Acetonitrile- $\left.d_{3}, 298 \mathrm{~K}\right)$ spectrum of $6 \mathbf{a}$. 


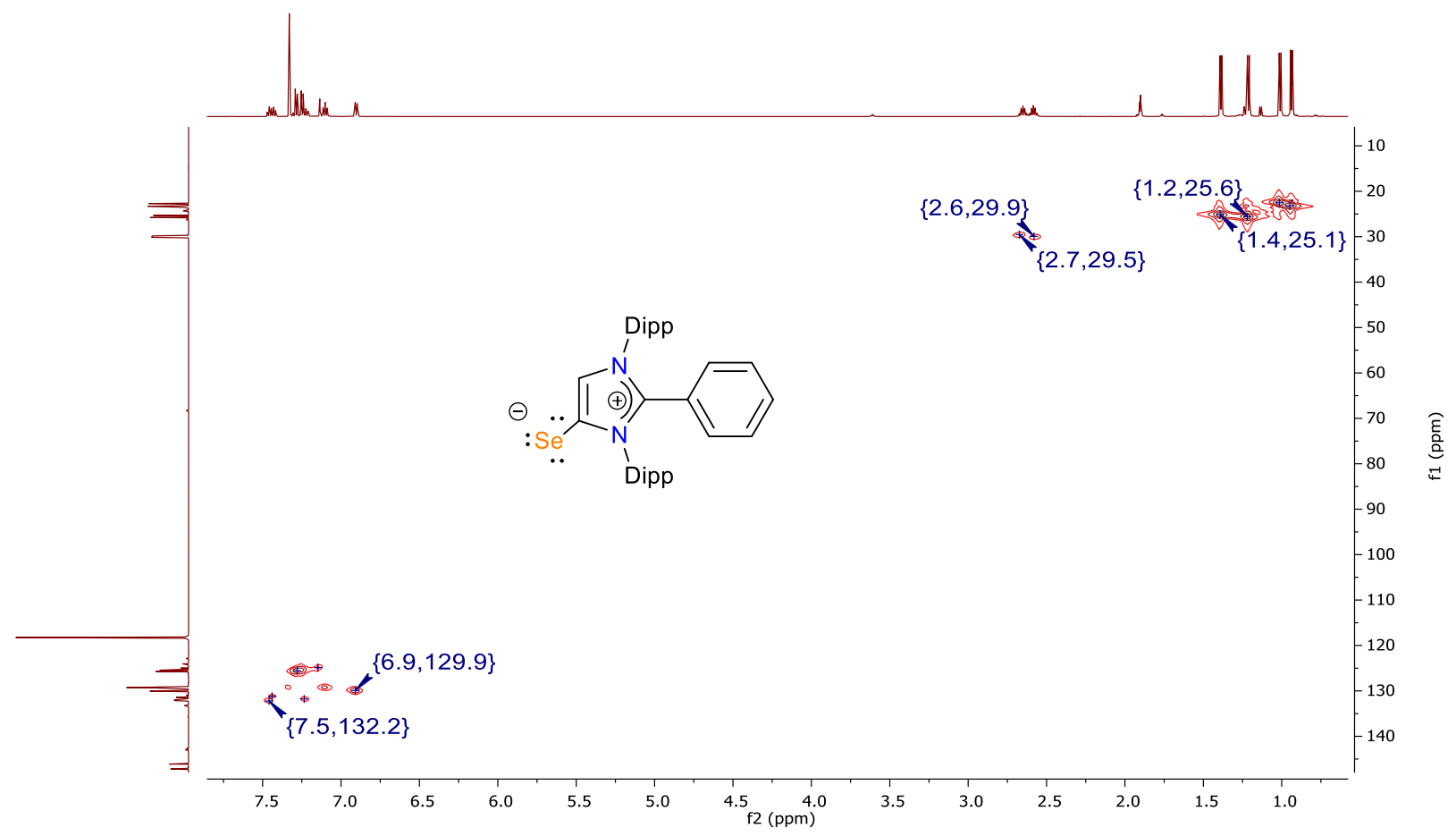

Figure S25. ${ }^{1} \mathrm{H}^{-13} \mathrm{C}$ HMQC NMR $\left(600 / 151 \mathrm{MHz}\right.$, Acetonitrile- $\left.d_{3}, 298 \mathrm{~K}\right)$ spectrum of $6 \mathbf{a}$.

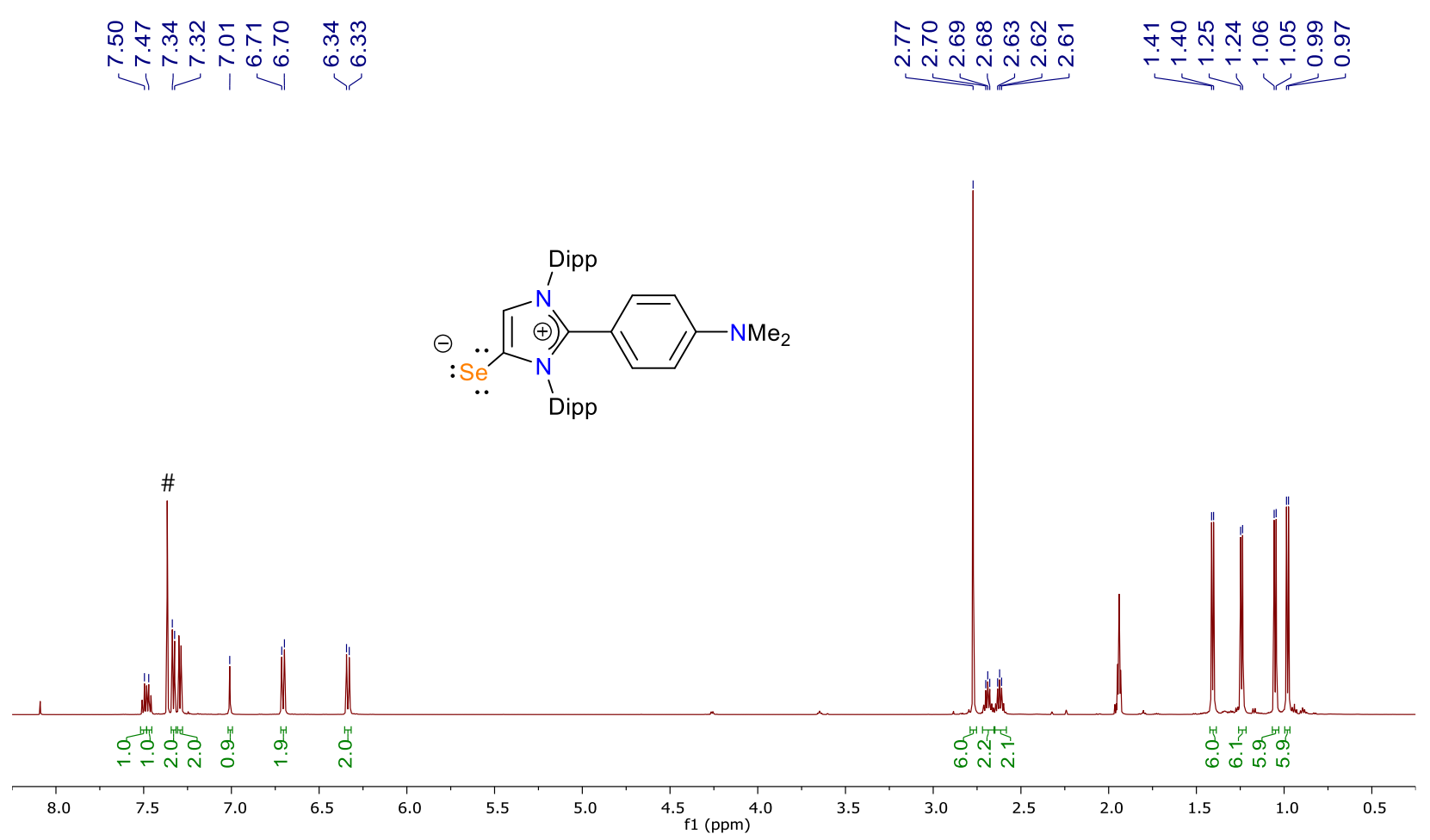

Figure S26. ${ }^{1} \mathrm{H}$ NMR (600 MHz, Acetonitrile- $\left.d_{3}, 298 \mathrm{~K}\right)$ spectrum of $6 \mathbf{b}$. (\# benzene) 


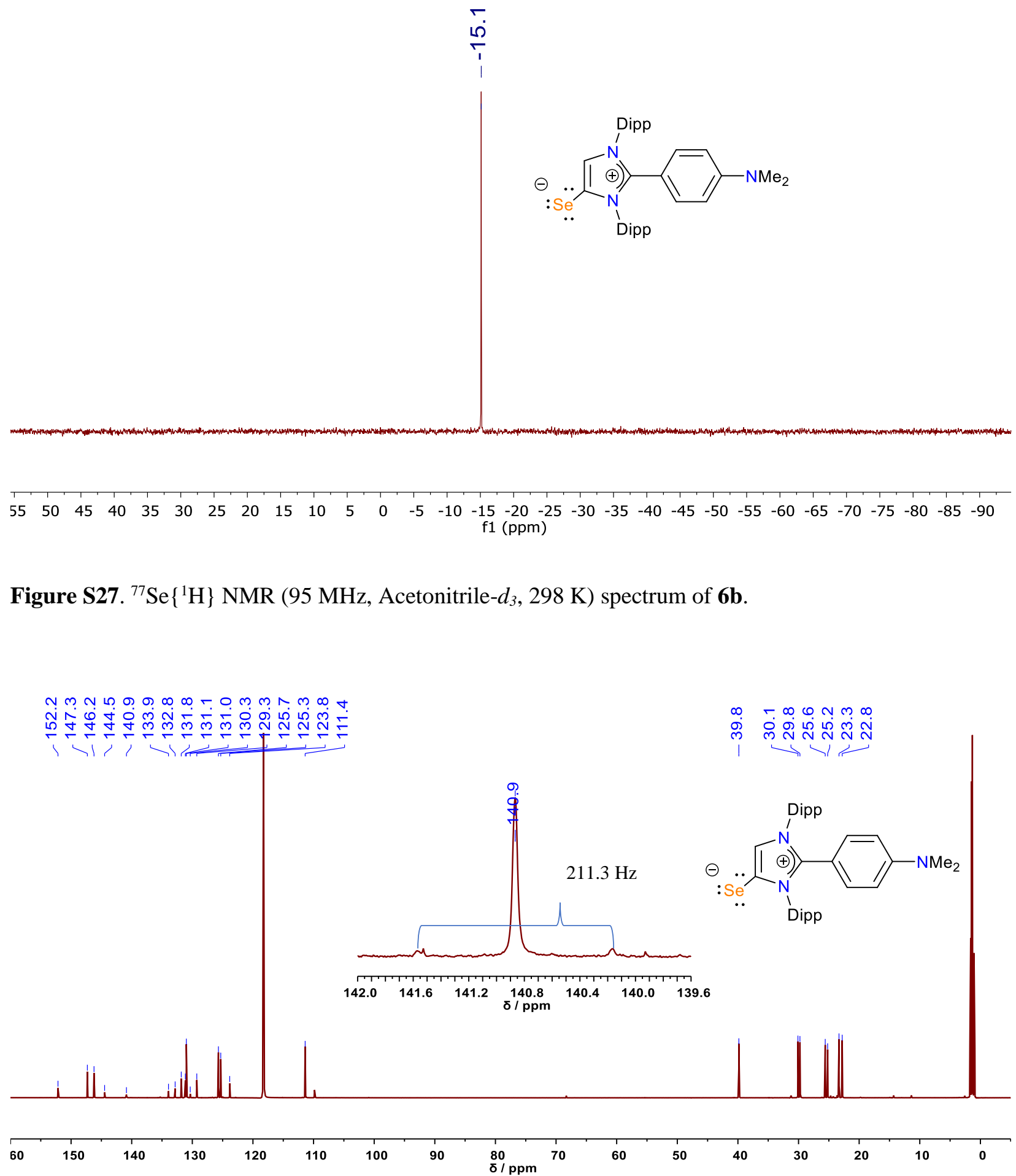

Figure S28. ${ }^{13} \mathrm{C}\left\{{ }^{1} \mathrm{H}\right\}$ NMR (151 MHz, Acetonitrile- $\left.d_{3}, 298 \mathrm{~K}\right)$ spectrum of $\mathbf{6 b}$. 


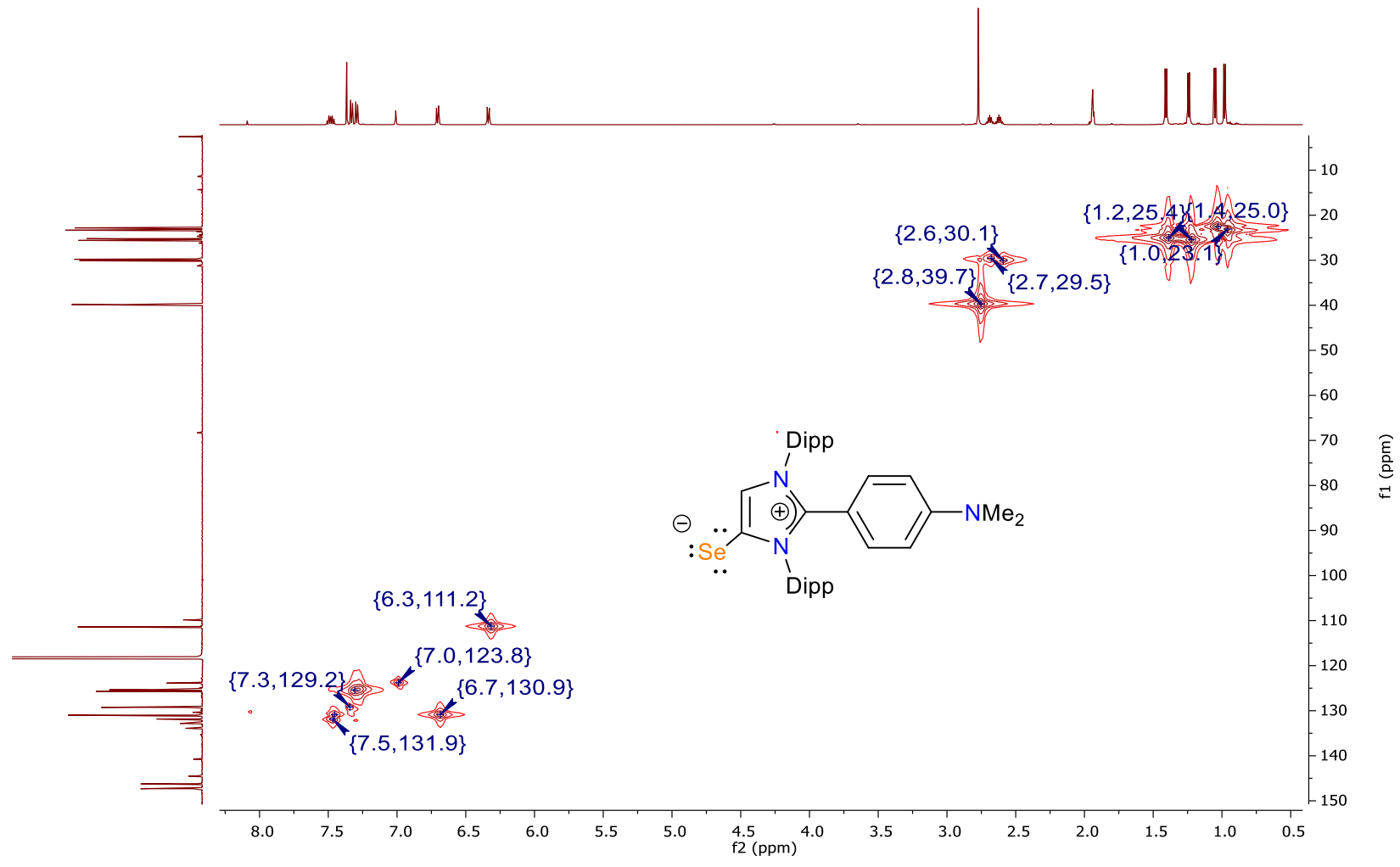

Figure S29. ${ }^{1} \mathrm{H}-{ }^{13} \mathrm{C}\left\{{ }^{1} \mathrm{H}\right\}$ NMR $\left(600 / 151 \mathrm{MHz}\right.$, Acetonitrile- $\left.d_{3}, 298 \mathrm{~K}\right)$ spectrum of $\mathbf{6 b}$.

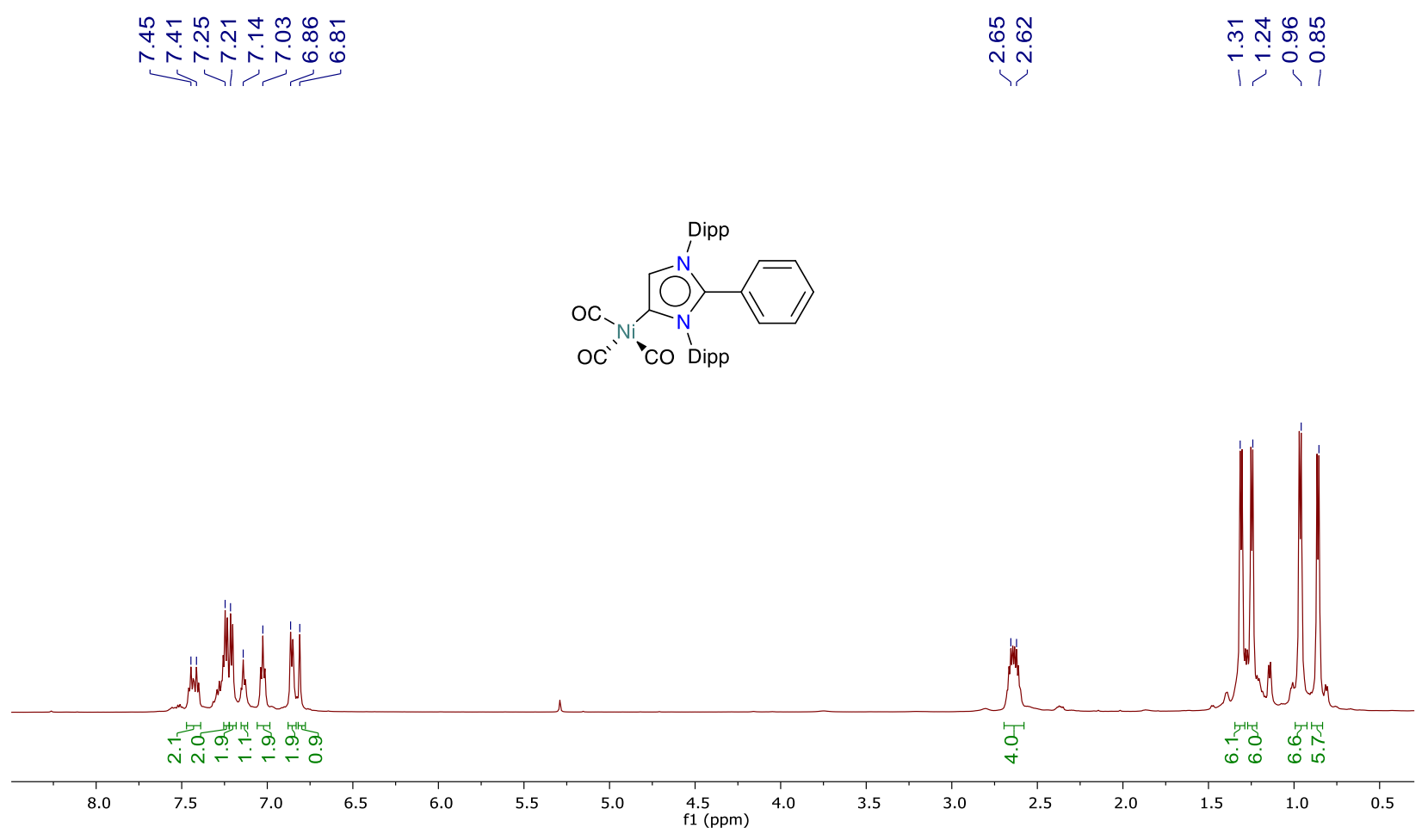

Figure S30. ${ }^{1} \mathrm{H}$ NMR $\left(600 \mathrm{MHz}, \mathrm{CDCl}_{3}, 298 \mathrm{~K}\right)$ spectrum of $7 \mathbf{a}$. 


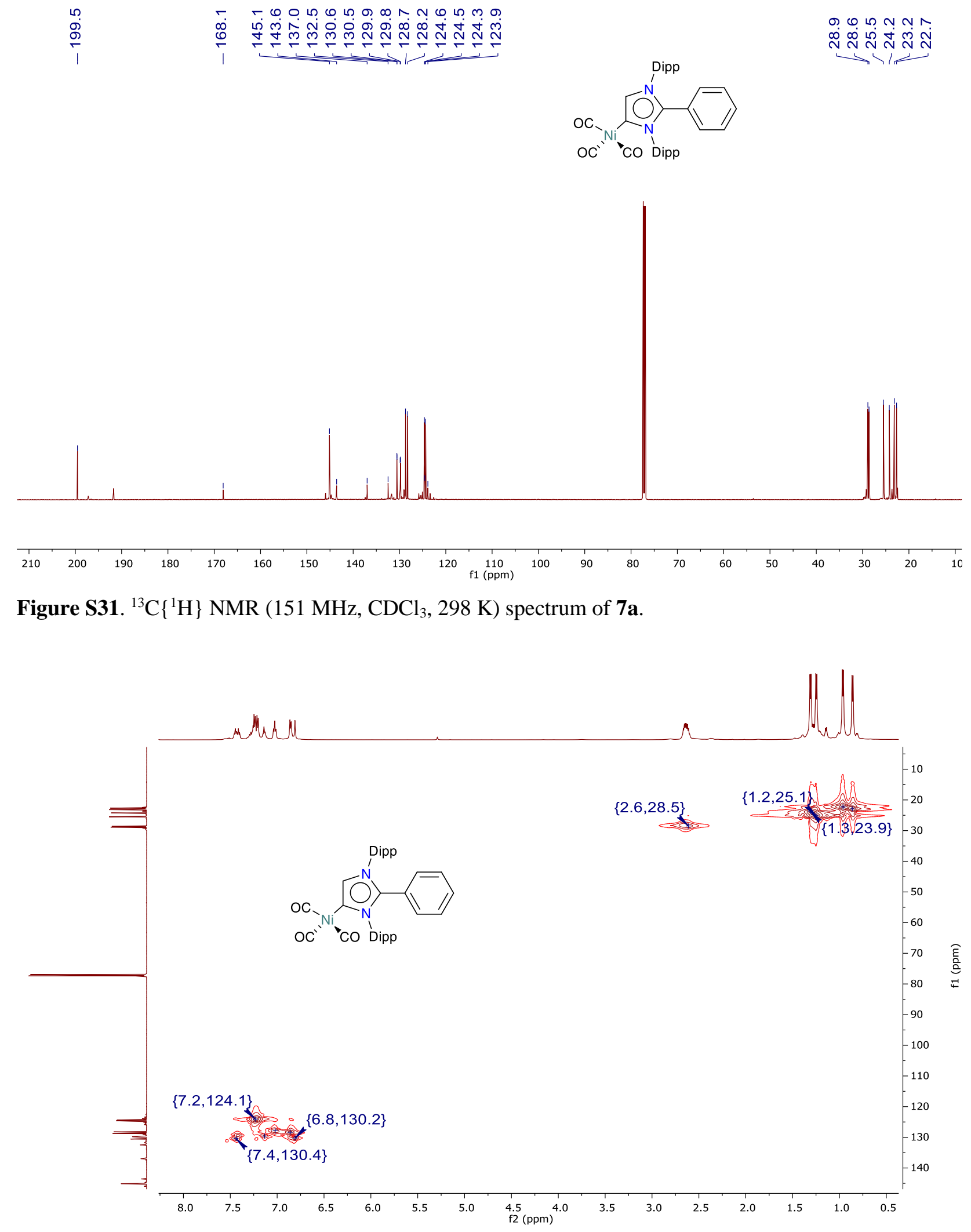

Figure S32. ${ }^{1} \mathrm{H}^{13} \mathrm{C}\left\{{ }^{1} \mathrm{H}\right\}$ NMR $\left(600 / 151 \mathrm{MHz}, \mathrm{CDCl}_{3}, 298 \mathrm{~K}\right)$ spectrum of 7a. 


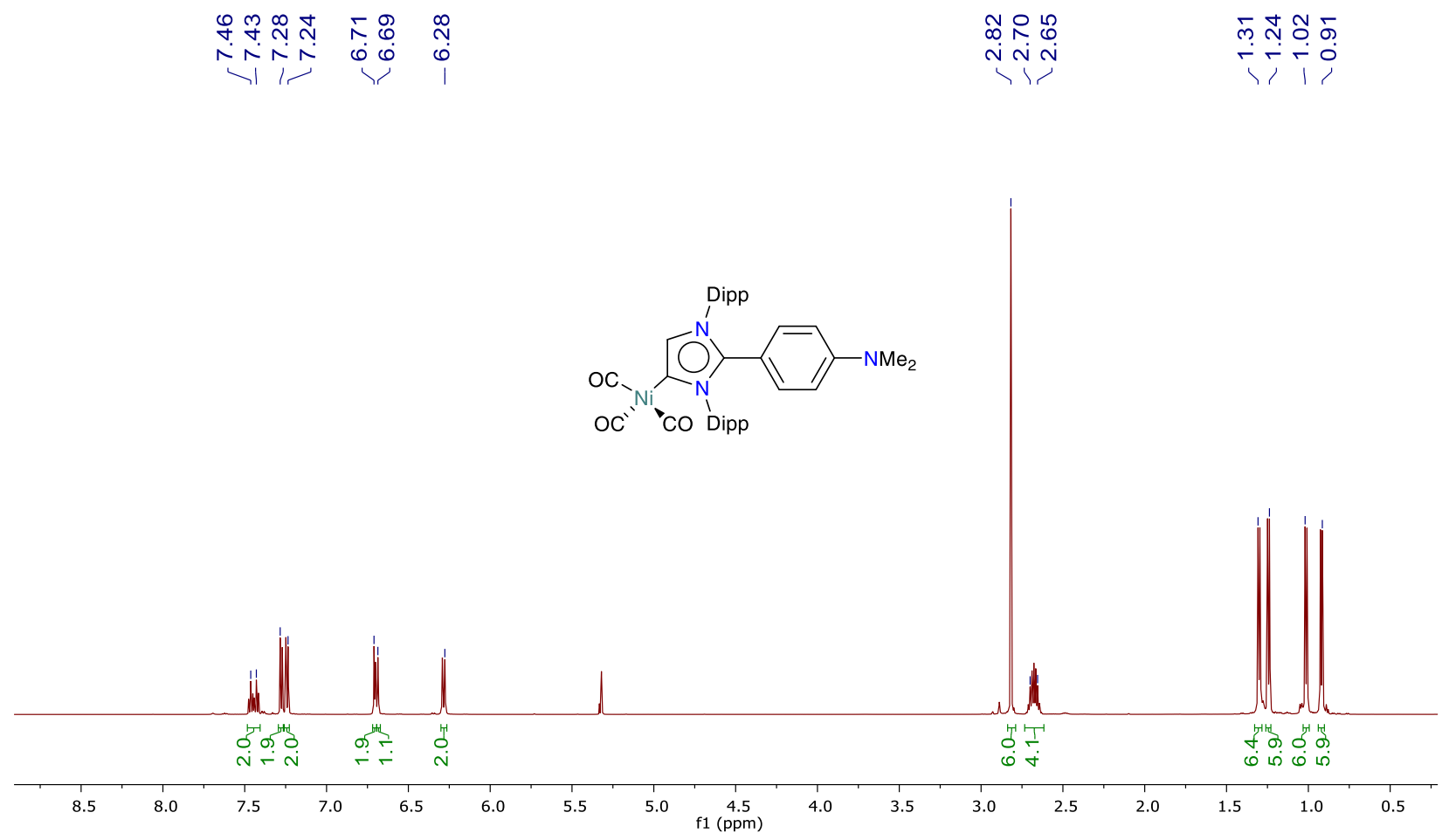

Figure S33. ${ }^{1} \mathrm{H}$ NMR $\left(600 \mathrm{MHz}, \mathrm{CD}_{2} \mathrm{Cl}_{2}, 298 \mathrm{~K}\right)$ spectrum of $7 \mathbf{b}$.

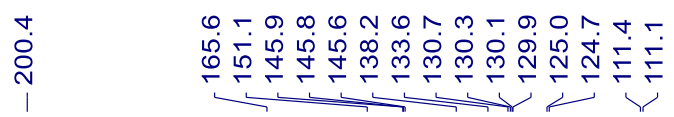

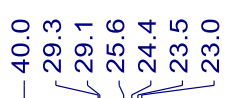
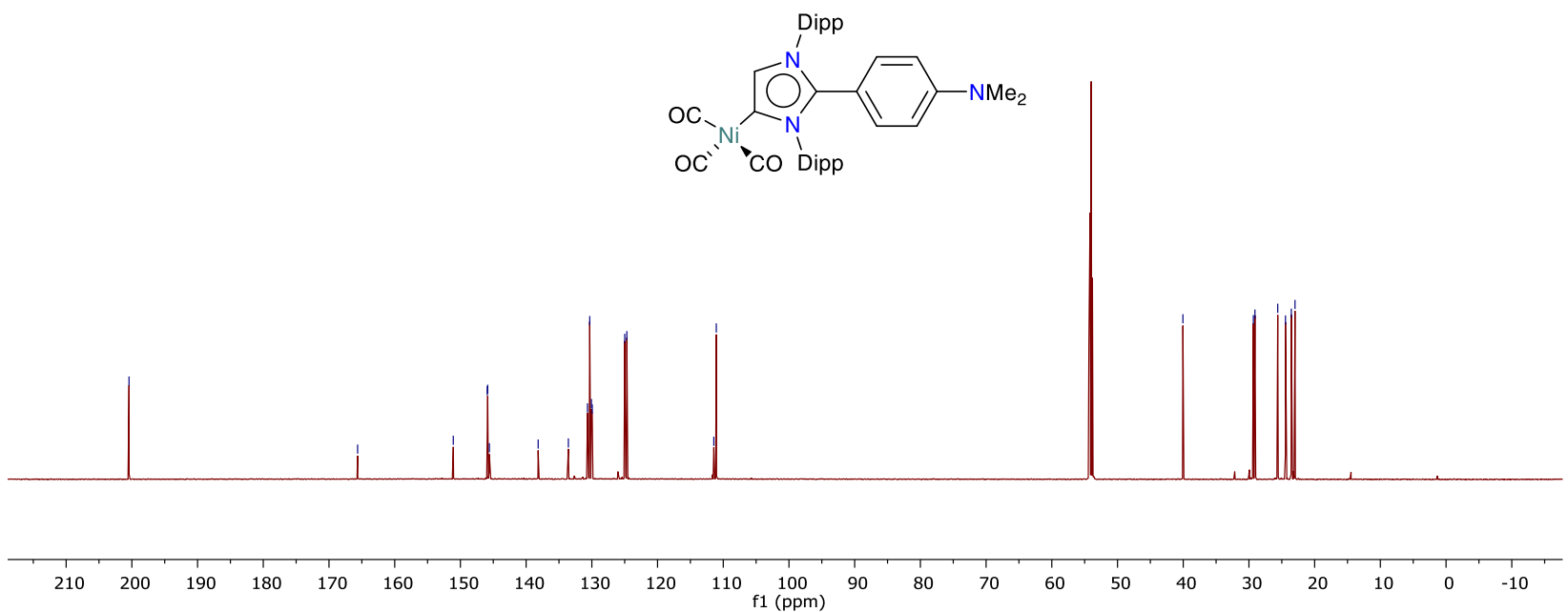

Figure S34. ${ }^{13} \mathrm{C}\left\{{ }^{1} \mathrm{H}\right\}$ NMR $\left(151 \mathrm{MHz}, \mathrm{CD}_{2} \mathrm{Cl}_{2}, 298 \mathrm{~K}\right)$ spectrum of $\mathbf{7 b}$. 


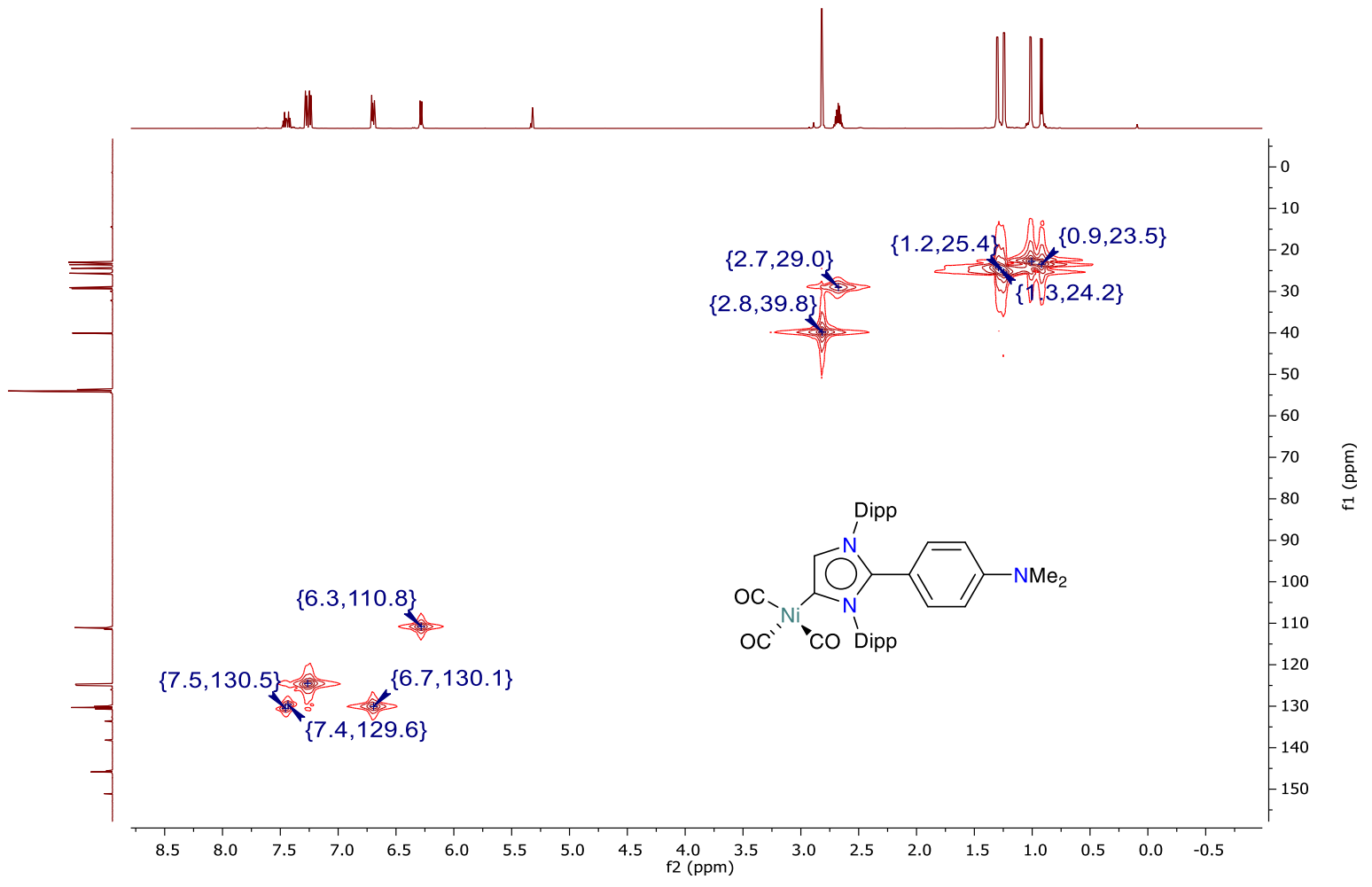

Figure S35. ${ }^{1} \mathrm{H}-{ }^{13} \mathrm{C}\left\{{ }^{1} \mathrm{H}\right\} \mathrm{NMR}\left(600 / 151 \mathrm{MHz}, \mathrm{CD}_{2} \mathrm{Cl}_{2}, 298 \mathrm{~K}\right)$ spectrum of $7 \mathbf{b}$. 
Plots of IR Spectra

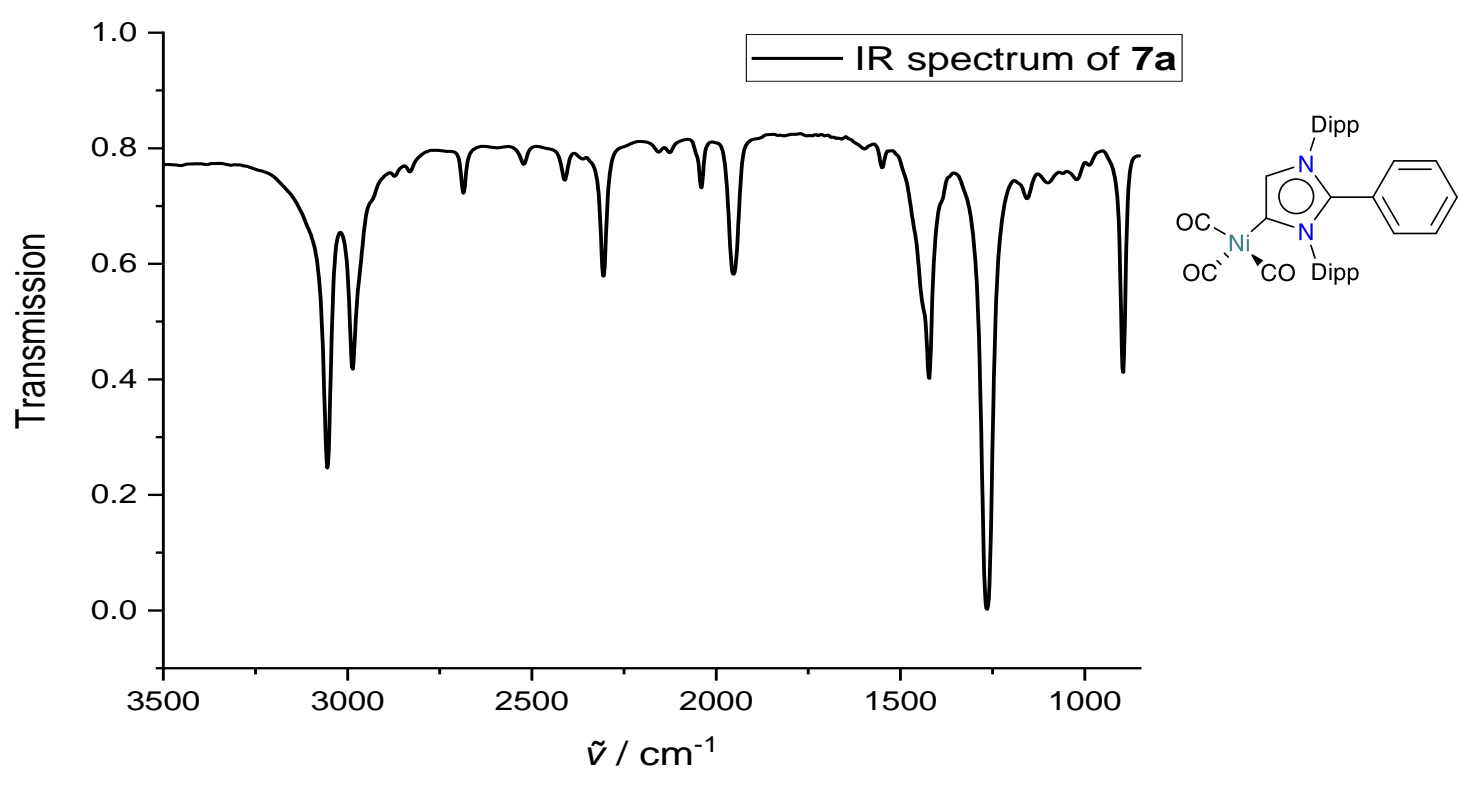

Figure S36. IR $\left(\mathrm{CH}_{2} \mathrm{Cl}_{2}, 298 \mathrm{~K}\right)$ spectrum of 7a.

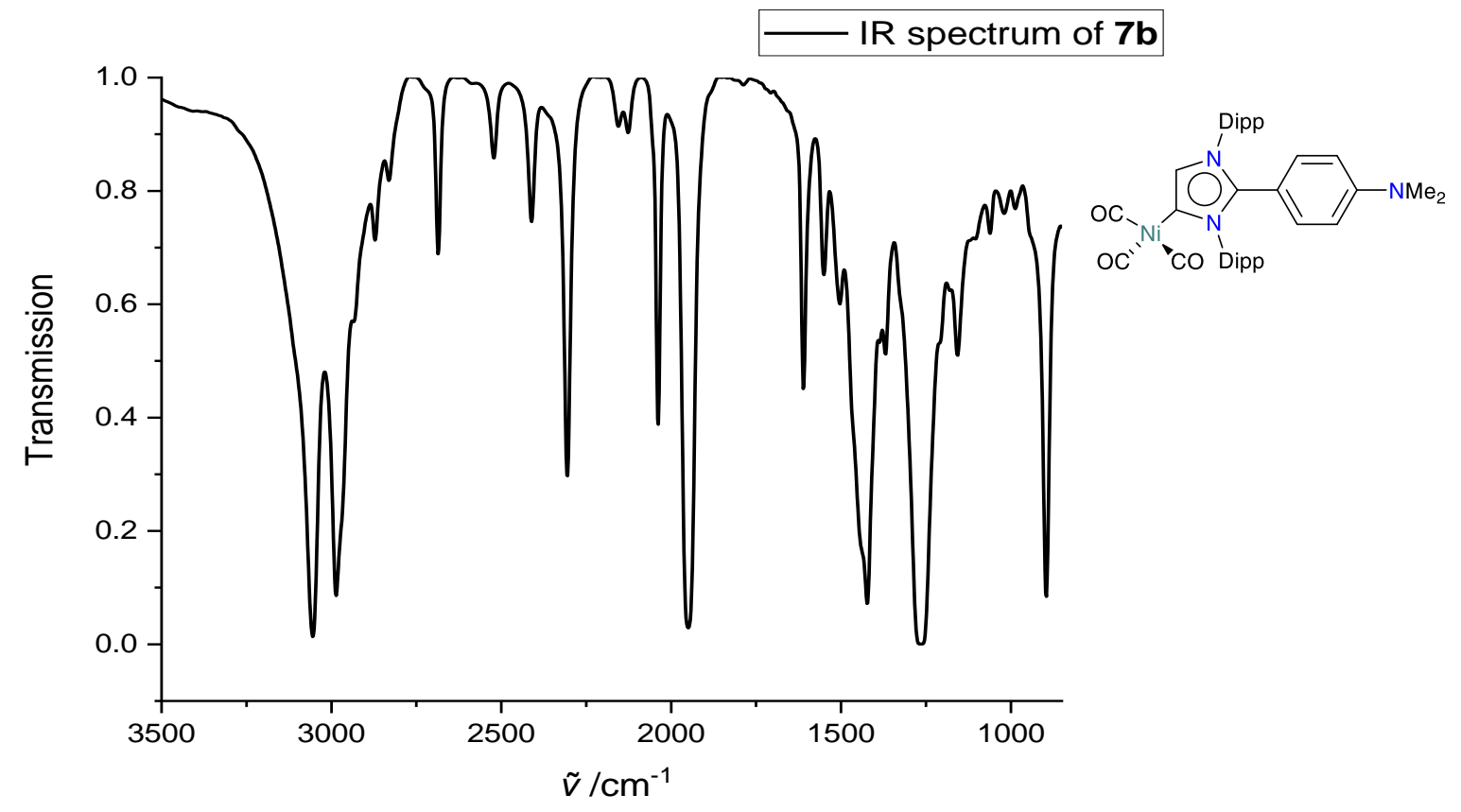

Figure S37. IR $\left(\mathrm{CH}_{2} \mathrm{Cl}_{2}, 298 \mathrm{~K}\right)$ spectrum of $\mathbf{7 b}$. 


\section{Crystallographic Details}

Single crystals were examined on a Rigaku Supernova diffractometer using $\mathrm{CuK} \alpha(\lambda=1.54184 \AA)$ or Mo $\mathrm{K} \alpha\left(\lambda=0.71073 \AA\right.$ A) radiation. Using Olex $2,{ }^{1}$ the structure was solved with the ShelXT ${ }^{2}(\mathbf{3 a}, \mathbf{4 a}, \mathbf{5 a}, \mathbf{6 a}, \mathbf{6 b}$, 7b) or ShelXS $S^{3}$ (7a) structure solution program using Intrinsic Phasing and refined with the ShelXL refinement package using Least Squares minimisation. One solvent THF molecule of 3a is disordered over two sites (78:22). Bond lengths were restrained with SADI, anisotropic displacement parameters with EADP. In the structure of $\mathbf{6 b}$, toluene is disordered over an inversion center. The structure of $7 \mathbf{a}$ shows a disorder of O1/C34 over two sites (63:37). 
Table S1. Crystallographic details of $\mathbf{3 a}, \mathbf{4 a}$ and $\mathbf{5 a}$

\begin{tabular}{|c|c|c|c|}
\hline & 3a(thf) & $4 \mathbf{a}$ & $5 \mathbf{5 a}$ \\
\hline Empirical formula & $\mathrm{C}_{40} \mathrm{H}_{57} \mathrm{IN}_{2} \mathrm{OSi}$ & $\mathrm{C}_{3}{ }_{9} \mathrm{H}_{45} \mathrm{ClIN}_{2} \mathrm{P}$ & $\mathrm{C}_{39} \mathrm{H}_{45} \mathrm{~N}_{2} \mathrm{P}$ \\
\hline Formula weight & 736.86 & 735.09 & 572.74 \\
\hline Temperature/K & $100.01(10)$ & $99.99(10)$ & $100.0(1)$ \\
\hline Crystal system & orthorhombic & triclinic & monoclinic \\
\hline Space group & Pna2 1 & P1 & $\mathrm{P} 21 / \mathrm{c}$ \\
\hline $\mathrm{a} / \AA ̊$ & $21.5136(2)$ & $9.7472(4)$ & $18.6617(5)$ \\
\hline $\mathrm{b} / \AA$ & $12.02290(10)$ & $9.7516(3)$ & $16.0128(5)$ \\
\hline $\mathrm{c} / \AA$ & $15.3752(2)$ & $10.8108(4)$ & $11.5543(3)$ \\
\hline$\alpha /^{\circ}$ & 90 & $70.928(3)$ & 90 \\
\hline$\beta /{ }^{\circ}$ & 90 & $67.704(4)$ & 103.666(3) \\
\hline$\gamma /{ }^{\circ}$ & 90 & $75.286(3)$ & 90 \\
\hline Volume $/ \AA^{3}$ & $3976.89(7)$ & $888.57(6)$ & $3354.97(18)$ \\
\hline $\mathrm{Z}$ & 4 & 1 & 4 \\
\hline$\rho_{\text {calc }} \mathrm{g} / \mathrm{cm}^{3}$ & 1.231 & 1.374 & 1.134 \\
\hline$\mu / \mathrm{mm}^{-1}$ & 0.866 & 1.050 & 0.925 \\
\hline $\mathrm{F}(000)$ & 1544.0 & 378.0 & 1232.0 \\
\hline Crystal size $/ \mathrm{mm}^{3}$ & $0.412 \times 0.251 \times 0.168$ & $0.153 \times 0.126 \times 0.015$ & $0.198 \times 0.113 \times 0.021$ \\
\hline Radiation/A & Mo $\mathrm{K} \alpha(\lambda=0.71073)$ & $\operatorname{MoK} \alpha(\lambda=0.71073)$ & $\mathrm{CuK} \alpha(\lambda=1.54184)$ \\
\hline $2 \Theta$ range for data collection $/^{\circ}$ & 5.082 to 65.688 & 4.214 to 64.28 & 7.366 to 149.86 \\
\hline Index ranges & $\begin{array}{c}-32 \leq \mathrm{h} \leq 32 \\
-18 \leq \mathrm{k} \leq 18 \\
-23 \leq 1 \leq 23\end{array}$ & $\begin{array}{l}-14 \leq \mathrm{h} \leq 14 \\
-14 \leq \mathrm{k} \leq 14 \\
-15 \leq 1 \leq 15\end{array}$ & $\begin{array}{l}-23 \leq \mathrm{h} \leq 20 \\
-18 \leq \mathrm{k} \leq 19 \\
-14 \leq 1 \leq 11\end{array}$ \\
\hline Reflections collected & 235566 & 26120 & 14436 \\
\hline Independent reflections & $\begin{array}{c}14370\left[R_{\text {int }}=0.0356,\right. \\
\left.R_{\text {sigma }}=0.0155\right]\end{array}$ & $\begin{array}{c}11145\left[R_{\text {int }}=0.0315,\right. \\
\left.R_{\text {sigma }}=0.0445\right]\end{array}$ & $\begin{array}{c}6712\left[\mathrm{R}_{\text {int }}=0.0561,\right. \\
\left.\mathrm{R}_{\text {sigma }}=0.0742\right]\end{array}$ \\
\hline Reflections with $I>2 \sigma(I)$ & 13009 & 10907 & 4707 \\
\hline Data/restraints/parameters & $14370 / 22 / 433$ & $11145 / 3 / 405$ & $6712 / 0 / 559$ \\
\hline Goodness-of-fit on $\mathrm{F}^{2}$ & 1.029 & 1.057 & 1.019 \\
\hline Final $\mathrm{R}$ indexes $[I>2 \sigma(I)]$ & $\mathrm{R}_{1}=0.0275, \mathrm{wR}_{2}=0.0630$ & $\mathrm{R}_{1}=0.0342, \mathrm{wR}_{2}=0.0775$ & $\mathrm{R}_{1}=0.0496, \mathrm{wR}_{2}=0.1102$ \\
\hline Final $\mathrm{R}$ indexes [all data] & $\mathrm{R}_{1}=0.0341, \mathrm{wR}_{2}=0.0662$ & $\mathrm{R}_{1}=0.0353, \mathrm{wR}_{2}=0.0781$ & $\mathrm{R}_{1}=0.0815, \mathrm{wR}_{2}=0.1257$ \\
\hline Largest diff. peak/hole / e $\AA^{-3}$ & $0.68 /-0.73$ & $1.61 /-0.44$ & $0.29 /-0.30$ \\
\hline Flack parameter & $-0.028(5)$ & $-0.023(6)$ & - \\
\hline CCDC number & 1964579 & 1964580 & 1964581 \\
\hline
\end{tabular}


Table S2. Crystallographic details of $\mathbf{6 a}, \mathbf{b}$ and $\mathbf{7 a}, \mathbf{b}$.

\begin{tabular}{|c|c|c|c|c|}
\hline & 6a(benzene) & $\mathbf{6 b}(0.5$ toluene $)$ & $7 \mathbf{a}$ & $7 \mathbf{b}$ \\
\hline Empirical formula & $\mathrm{C}_{39} \mathrm{H}_{46} \mathrm{~N}_{2} \mathrm{Se}$ & $\mathrm{C}_{38.5} \mathrm{H}_{49} \mathrm{~N}_{3} \mathrm{Se}$ & $\mathrm{C}_{36} \mathrm{H}_{40} \mathrm{~N}_{2} \mathrm{NiO}_{3}$ & $\mathrm{C}_{38} \mathrm{H}_{45} \mathrm{~N}_{3} \mathrm{NiO}_{3}$ \\
\hline Formula weight & 621.74 & 632.76 & 607.41 & 650.48 \\
\hline Temperature/K & $95.00(10)$ & $100.00(14)$ & $100.01(10)$ & $100.00(10)$ \\
\hline Crystal system & monoclinic & monoclinic & orthorhombic & monoclinic \\
\hline Space group & $\mathrm{P} 21 / \mathrm{n}$ & $\mathrm{C} 2 / \mathrm{c}$ & Pbca & $\mathrm{P} 21 / \mathrm{n}$ \\
\hline $\mathrm{a} / \AA ̊ A$ & $14.70494(15)$ & $17.0091(4)$ & $11.56704(18)$ & $11.90787(13)$ \\
\hline $\mathrm{b} / \AA$ & $11.66663(12)$ & $17.9048(3)$ & $19.9683(3)$ & $23.4733(2)$ \\
\hline $\mathrm{c} / \AA ̊ \AA$ & $19.8031(3)$ & $24.1028(5)$ & $27.8938(5)$ & $12.98965(15)$ \\
\hline$\beta /^{\circ}$ & $94.2626(10)$ & $107.423(2)$ & 90 & $103.6178(11)$ \\
\hline Volume $/ \AA^{3}$ & $3387.97(7)$ & $7003.6(3)$ & $6442.76(18)$ & $3528.75(7)$ \\
\hline $\mathrm{Z}$ & 4 & 8 & 8 & 4 \\
\hline$\rho_{\text {calc }} / \mathrm{cm}^{3}$ & 1.219 & 1.200 & 1.252 & 1.224 \\
\hline$\mu / \mathrm{mm}^{-1}$ & 1.137 & 1.101 & 1.152 & 0.588 \\
\hline $\mathrm{F}(000)$ & 1312.0 & 2680.0 & 2576.0 & 1384.0 \\
\hline Crystal size $/ \mathrm{mm}^{3}$ & $0.437 \times 0.326 \times 0.227$ & $0.322 \times 0.259 \times 0.196$ & $0.325 \times 0.17 \times 0.051$ & $0.475 \times 0.306 \times 0.114$ \\
\hline Radiation/Å & $\operatorname{MoK} \alpha(\lambda=0.71073)$ & Mo K $\alpha(\lambda=0.71073)$ & $\mathrm{Cu} \mathrm{K} \alpha(\lambda=1.54184)$ & $\operatorname{MoK} \alpha(\lambda=0.71073)$ \\
\hline $2 \Theta$ range for data collection $/^{\circ}$ & 3.334 to 64.31 & 3.388 to 64.32 & 6.338 to 153.804 & 3.47 to 64.504 \\
\hline Index ranges & $\begin{array}{r}-22 \leq \mathrm{h} \leq 21 \\
-16 \leq \mathrm{k} \leq 17 \\
-29 \leq 1 \leq 28\end{array}$ & $\begin{array}{r}-23 \leq \mathrm{h} \leq 25 \\
-26 \leq \mathrm{k} \leq 26 \\
-35 \leq 1 \leq 34\end{array}$ & $\begin{array}{r}-14 \leq \mathrm{h} \leq 14 \\
-20 \leq \mathrm{k} \leq 25 \\
-33 \leq 1 \leq 35\end{array}$ & $\begin{array}{r}-17 \leq \mathrm{h} \leq 17 \\
-34 \leq \mathrm{k} \leq 35 \\
-19 \leq 1 \leq 19\end{array}$ \\
\hline Reflections collected & 67428 & 66658 & 53247 & 141167 \\
\hline Independent reflections & $\begin{array}{c}11254\left[\mathrm{R}_{\text {int }}=0.0348,\right. \\
\left.\mathrm{R}_{\text {sigma }}=0.0262\right]\end{array}$ & $\begin{array}{c}11518\left[\mathrm{R}_{\text {int }}=0.0394\right. \\
\left.\mathrm{R}_{\text {sigma }}=0.0288\right]\end{array}$ & $\begin{array}{c}6743\left[\mathrm{R}_{\text {int }}=0.0491\right. \\
\left.\mathrm{R}_{\text {sigma }}=0.0241\right]\end{array}$ & $\begin{array}{c}12008\left[\mathrm{R}_{\text {int }}=0.0357\right. \\
\left.\mathrm{R}_{\text {sigma }}=0.0179\right]\end{array}$ \\
\hline Reflections with $I>2 \sigma(I)$ & 9586 & 9771 & 5881 & 10521 \\
\hline Data/restraints/parameters & $11254 / 0 / 387$ & $11518 / 0 / 425$ & $6743 / 1 / 394$ & $12008 / 0 / 416$ \\
\hline Goodness-of-fit on $\mathrm{F}^{2}$ & 1.036 & 1.056 & 1.039 & 1.082 \\
\hline Final $\mathrm{R}$ indexes $[I>2 \sigma(I)]$ & $\mathrm{R}_{1}=0.0317, \mathrm{wR}_{2}=0.0697$ & $\begin{array}{c}\mathrm{R}_{1}=0.0394, \mathrm{wR} \mathrm{R}_{2}= \\
0.0980\end{array}$ & $\begin{array}{c}\mathrm{R}_{1}=0.0438, w \mathrm{R}_{2}= \\
0.1200\end{array}$ & $\begin{array}{c}\mathrm{R}_{1}=0.0362, \mathrm{wR}_{2}= \\
0.0913\end{array}$ \\
\hline Final $\mathrm{R}$ indexes [all data] & $\mathrm{R}_{1}=0.0419, \mathrm{wR}_{2}=0.0734$ & $\begin{array}{c}\mathrm{R}_{1}=0.0503, \mathrm{wR}_{2}= \\
0.1036\end{array}$ & $\begin{array}{c}\mathrm{R}_{1}=0.0504, w \mathrm{R}_{2}= \\
0.1257\end{array}$ & $\begin{array}{c}\mathrm{R}_{1}=0.0438, \mathrm{wR}_{2}= \\
0.0950\end{array}$ \\
\hline Largest diff. peak/hole / e $\AA^{-3}$ & $0.71 /-0.65$ & $1.48 /-0.65$ & $0.50 /-0.68$ & $0.54 /-0.54$ \\
\hline CCDC number & 1964582 & 1964583 & 1964584 & 1964585 \\
\hline
\end{tabular}




\section{Steric Maps of (iMIC $\left.{ }^{\mathrm{Ph}}\right) \mathrm{CuI}(8 \mathrm{a})$ and $\left(\mathrm{iMIC} \mathrm{CMP}^{\mathrm{DMP}}\right) \mathrm{CuBr}(8 \mathrm{~b})$}

The buried volume and steric map of copper complexes $\mathbf{8 a}$ and $\mathbf{8 b}$ were obtained using the SabmVca 2.1 web application. ${ }^{5}$

\section{Input data for 8a:}

Bondi radii scaled by 1.17

Sphere radius: $3.5 \AA$

Distance of the coordination point from the center of the sphere: $0.10 \AA$ Mesh spacing for numerical integration: 0.10

Hydrogens not included

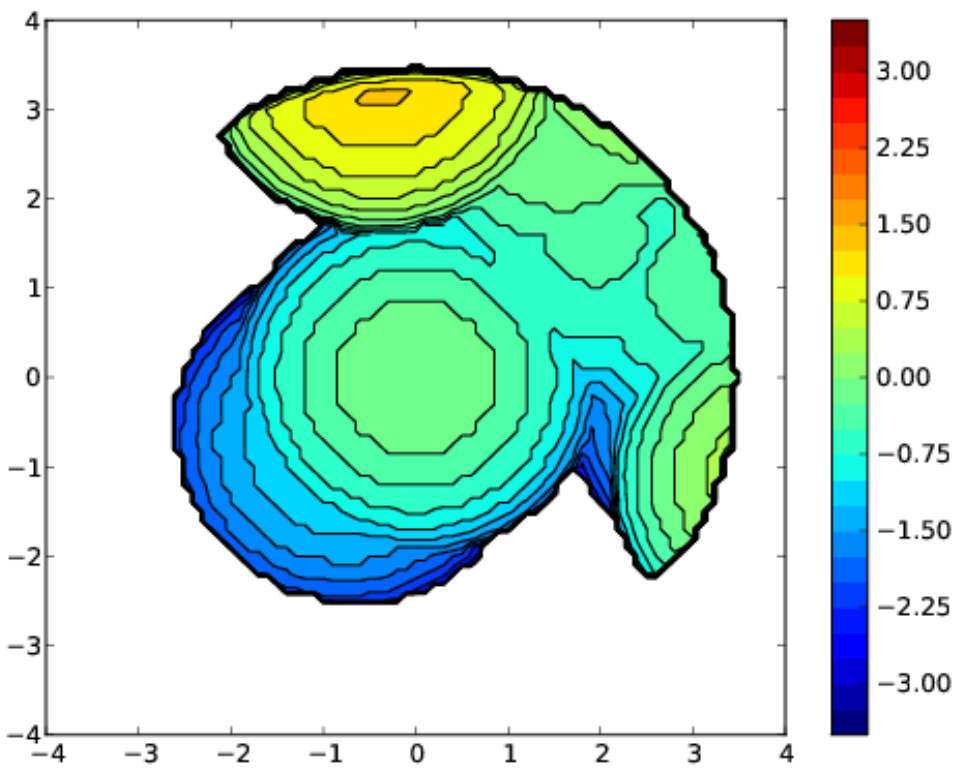

Figure S38. Steric map of compound 8a.

\section{Input data for $8 \mathrm{~b}$ :}

Bondi radii scaled by 1.17

Sphere radius: $3.5 \AA$

Distance of the coordination point from the center of the sphere: $0.11 \AA$

Mesh spacing for numerical integration: 0.10

Hydrogens not included

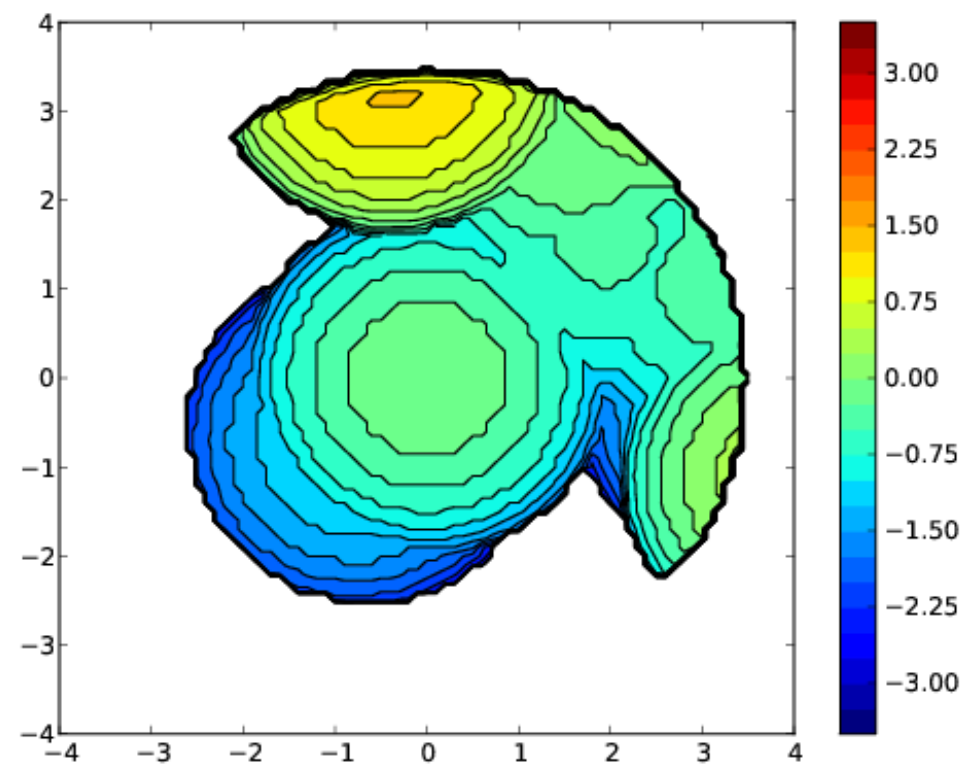

Figure S39. Steric map of compound $\mathbf{8 b}$. 


\section{Correlation plots of ${ }^{31} \mathrm{P}$ vs. ${ }^{77} \mathrm{Se}$ NMR chemical shifts}

Fit function for exponential correlation:

$$
\delta\left({ }^{31} P\right)=114.53453-162.35007 \cdot e^{-0.00193 \cdot \delta\left({ }^{77} S e\right)}, R^{2}=0.97027
$$

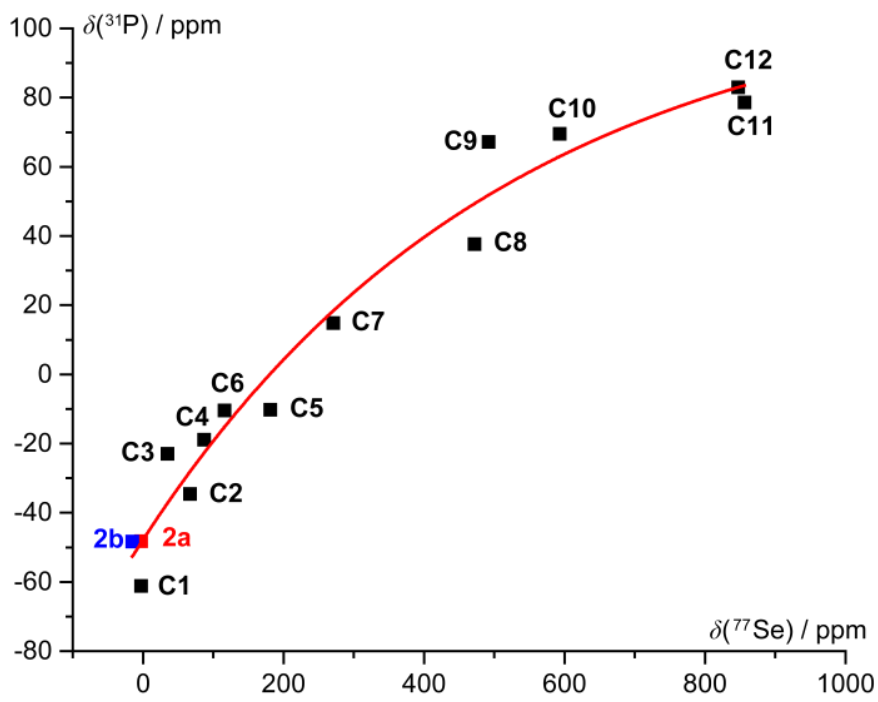

Figure S40: $\quad$ Plot of ${ }^{31} \mathrm{P}$ against ${ }^{77} \mathrm{Se}$ NMR chemical shifts of representative $(\mathbf{C}) \mathrm{PPh}$ and $(\mathbf{C}) \mathrm{Se}$ compounds $(\mathbf{C}=$ singlet carbene $)$ with an exponential fit function.

Fit function for linear correlation:

$$
\delta\left({ }^{31} P\right)=0.15842 \cdot \delta\left({ }^{77} S e\right)-38.35937, R^{2}=0.92704
$$

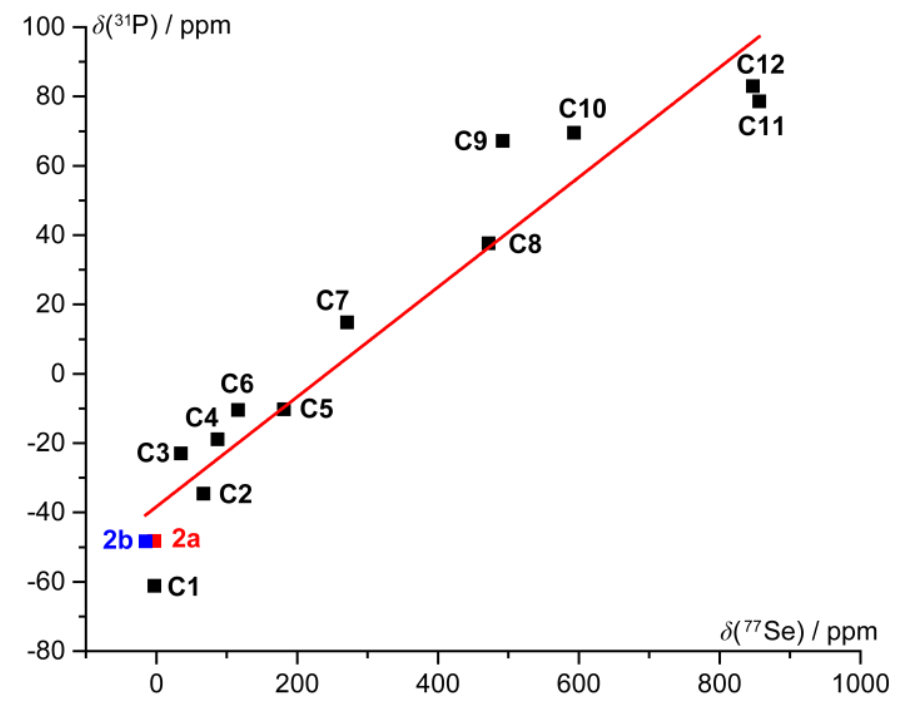

Figure S41: $\quad$ Plot of ${ }^{31} \mathrm{P}$ against ${ }^{77} \mathrm{Se}$ NMR chemical shifts of representative $(\mathbf{C}) \mathrm{PPh}$ and $(\mathbf{C}) \mathrm{Se}$ compounds $(\mathbf{C}=$ singlet carbene $)$ with a linear fit function. 


\section{References}

1. O. V. Dolomanov, L. J. Bourhis., R. J. Gildea, J. A. K. Howard, H. Puschmann, J. Appl. Cryst. 2009, 42, 339-341.

2. G. M. Sheldrick, Acta Cryst. A 2015, 71, 3-8.

3. G. M. Sheldrick, Acta Cryst. A 2008, 64, 112-122.

4. G. M. Sheldrick, Acta Cryst. C 2015, 71, 3-8.

5. L. Falivene, Z. Cao, A. Petta, L. Serra, A. Poater, R. Oliva, V. Scarano, L. Cavallo, Nat. Chem. 2019, 11, 872-879. 